\title{
1 Focal adhesion-generated cues in extracellular matrix regulate 2 cell migration by local induction of clathrin-coated plaques
}

3 Delia Bucher ${ }^{1,2,6}$, Markus Mukenhirn ${ }^{1,2}$, Kem A. Sochacki ${ }^{3}$, Veronika Saharuka ${ }^{1,2}$, Christian

4 Huck $^{4}$, Chiara Zambarda5,6, Justin W. Taraska3 ${ }^{3}$ Elisabetta Ada Cavalcanti-Adam5,6, Steeve

5 Boulant ${ }^{1,2, \#}$

61 Department of Infectious Diseases, Virology, University Hospital Heidelberg, Im 7 Neuenheimer Feld 344, 69120 Heidelberg, Germany

82 German Cancer Research Center (DKFZ), Im Neuenheimer Feld 581, 69120 Heidelberg, 9 Germany

$10{ }^{3}$ National Heart Lung and Blood Institute, National Institutes of Health, Bethesda, MD,

1120892, USA

124 Kirchhoff Institute for Physics, Heidelberg University, Im Neuenheimer Feld 227, 69120

13 Heidelberg, Germany

145 Max Planck Institute for Medical Research, D-69120 Heidelberg, Germany

$15{ }^{6}$ Heidelberg University, D-69120 Heidelberg, Germany

16 \#Correspondence should be addressed to Steeve Boulant (s.boulant@dkfz.de) 


\section{Abstract}

19 Clathrin is a unique scaffold protein, which forms polyhedral lattices with flat and curved

20 morphology. The function of curved clathrin-coated pits in forming endocytic structures is

21 well studied. On the contrary, the role of large flat clathrin arrays, called clathrin-coated

22 plaques, remains ambiguous. Previous studies suggested an involvement of plaques in cell

23 adhesion. However, the molecular origin leading to their formation and their precise

24 functions remain to be determined. Here, we study the origin and function of clathrin-

25 coated plaques during cell migration. We revealed that plaque formation is intimately

26 linked to extracellular matrix (ECM) modification by focal adhesions (FAs). We show that in

27 migrating cells, FAs digest the ECM creating extracellular topographical cues that dictate the

28 future location of clathrin-coated plaques. We identify Eps15 and Eps15R as key regulators

29 for the formation of clathrin-coated plaques at locally remodelled ECM sites. Using a genetic

30 silencing approach to abrogate plaque formation and 3D-micropatterns to spatially control

31 the location of clathrin-coated plaques, we could directly correlate cell migration

32 directionality with the formation of clathrin-coated plaques and their ability to recognize

33 extracellular topographical cues. We here define the molecular mechanism regulating the

34 functional interplay between FAs and plaques and propose that clathrin-coated plaques act

35 as regulators of cell migration promoting contact guidance-mediated collective migration in

36 a cell-to-cell contact independent manner. 


\section{Introduction}

39 Each cell is surrounded by a plasma membrane composed of lipids that separates the 40 intracellular milieu from the extracellular space. To sense and interact with the 41 extracellular environment, cells exploit both integral and peripheral membrane proteins 42 and establish defined domains at the plasma membrane to mediate processes like 43 signalling, endocytosis and mechanotransduction.

44 The main component of the environment surrounding cells is the extracellular matrix 45 (ECM), a complex network of secreted macromolecules such as collagen, laminin and 46 fibronectin ${ }^{1,2}$. Specific binding sites on these proteins serve as ligands for cellular 47 receptors ${ }^{3-5}$. The most important of adhesion receptors are integrins, which, together with 48 adaptors, scaffold and signalling proteins, assemble into the multi-layered adhesive unit 49 referred to as focal adhesions (FAs) 3,6,7. FAs mediate adhesion and signalling between the 50 inside and the outside of the cell7,8. They are also viewed as mechanotransducing units 51 coupling biophysical properties of the ECM to intracellular processes ${ }^{8}$. Indeed, not only the 52 chemical but also the topographical and mechanical properties of the ECM influence cellular 53 functions such as guiding cell migration ${ }^{9,10}$ and maintaining stem cell niches ${ }^{11,12}$. The ECM is 54 not a static scaffold; it is a highly dynamic mesh constantly renewed and remodelled by 55 cells, which in return react to the new properties of their surrounding matrix ${ }^{13,14}$. ECM 56 remodelling is achieved both though cellular-mediated physical forces ${ }^{15}$ as well as through 57 enzymatic digestions by specific enzymes like the matrix metalloproteinases (MMPs) ${ }^{13}$.

58 Beside FA, other plasma membrane-associated supramolecular complexes have been 59 described to have mechanotransduction properties e.g. podosomes ${ }^{16}$ and cadherin60 mediated cell-cell junctions ${ }^{17}$. Recently, plasma membrane-associated clathrin structures 61 have been shown to assemble at specific plasma membrane sites in response to unique 62 topographical profiles and mechanical properties of the extracellular environment18-21. 63 Together with numerous adaptors and accessory proteins, clathrin molecules assemble at 64 the plasma membrane to form a highly dynamic array 22,23 . During clathrin-mediated 65 endocytosis (CME), small transient clathrin coats can form initially as curved or flat arrays 66 which will rearrange to form clathrin-coated pits (CCPs) with a typical diameter of 100-150 $67 \mathrm{~nm}^{24-26}$. Distinct from these small endocytic structures, flat long-lived larger clathrin coats, 68 known as clathrin-coated plaques, are frequently observed ${ }^{27}$. These clathrin-coated plaques 
69 appear to have pleiotropic functions. They can facilitate endocytosis and signalling via 70 clustering of plasma membrane receptors and nucleating endocytic events ${ }^{28-30}$. The putative

71 role of larger clathrin arrays in cell adhesion and migration has long been discussed ${ }^{31,32}$. It

72 has recently been readdressed with the observation that specialized clathrin arrays named 73 tubular clathrin/AP2 lattices (TCALs) are responsible for binding collagen fibres in a 3D-

74 environment ${ }^{19}$ and that association of clathrin-coated plaques with the ECM is integrin 75 dependent $27,33,34$.

76 To date, the mechanisms that lead to clathrin-coated plaque formation and stabilization at

77 the plasma membrane and the cellular and extracellular determinants that dictate whether 78 a clathrin-coated plaque displays an endocytic or a non-endocytic function are still unclear.

79 Similarly, the molecular and/or physical determinants that drive clathrin-coated plaque

80 formation remain poorly understood. There is evidence that some clathrin-coated plaques

81 are resulting from frustrated endocytic events ${ }^{33}$. However, it remains unclear whether cells

82 can generate specific cellular and/or extracellular signals that lead to a local induction of

83 clathrin-coated plaques which in turn will provide the cell with a specific function.

84 In this work, we demonstrate that disassembling FAs, at the leading edge of the cell, are 85 replaced by clathrin-coated plaques during cell migration, in an integrin-dependent process,

86 that we term "switch from FAs to clathrin-coated plaques". We could trace the signals 87 leading to this switch back to the digestion of the ECM and the generation of extracellular 88 topographical cues by FAs. We identified Eps15 and Eps15R as important players in the 89 formation of clathrin-coated plaques. Migration assays of wild type and Eps15/R depleted 90 cells, allowed us to correlate directionality of cell migration with the formation of clathrin91 coated plaques at topographical cues. This work reveals a novel function of clathrin-coated 92 plaques and demonstrates that flat clathrin arrays act as plasma membrane-associated 93 supramolecular complexes which sense extracellular topographical cues to influence cell 94 behaviour. 


\section{Results}

\section{Dynamics and ultrastructural characterization of clathrin-coated plaques}

98 The presence of clathrin-coated plaques at the plasma membrane has been long known 31,35 ,

99 but their function is still poorly understood. It was shown that their presence was cell type 100 specific and that they were limited to the ventral plasma membrane ${ }^{36}$. This side-specific 101 localization and the fact that clathrin-coated plaques are in close contact with the 102 substrate ${ }^{31,32}$ as well as associated with the ECM receptors integrins ${ }^{27,32-34}$ strongly suggest 103 a function in cell adhesion. Aiming at characterizing this function, we exploited the human 104 glioblastoma U373 cell line previously reported to display clathrin-coated plaques ${ }^{36,37}$. Live 105 fluorescence microscopy of U373 stably expressing the sigma subunit of the clathrin 106 adaptor AP2 fused to eGFP (AP2-eGFP) showed clathrin structures with high fluorescence 107 intensity and a long lifetime as well as non-terminated events (Supplementary Fig. 1a). In 108 contrast, transient endocytic clathrin structures had both a lower fluorescent intensity and 109 lifetime. Transmission electron microscopy (TEM) of metal replicas confirmed the presence 110 of flat clathrin-coated plaques with or without budding CCPs at the rim, displaying a surface 111 area of up to $100,000 \mathrm{~nm}^{2}$. In comparison, the endocytic invaginated spherical CCPs display 112 an average projected area of $15,000 \mathrm{~nm}^{2}$ (Supplementary Fig. 1b-e). Correlative light and 113 electron microscopy (CLEM) of these U373 cells expressing AP2-eGFP confirmed that the 114 high fluorescence long-lived clathrin-coated structures (Supplementary Fig. 1a) were 115 indeed clathrin-coated plaques (Supplementary Fig. 1f-g). As such, in this work, we will 116 define clathrin-coated plaques as long-lived clathrin coats using live-cell fluorescence 117 microscopy. We could confirm that these structures are exclusively found at the ventral side 118 of cells (Supplementary Fig. 2a-b) and furthermore, show that this side specific localization 119 of clathrin-coated plaques correlates with the interaction of the cell with the extracellular 120 environment. Cells grown on adhesive micropatterned substrates display both clathrin121 coated plaques and CCPs on the adhesive parts whereas only CCPs are observed on the non122 adhesive sections of the substrate (Supplementary Fig. 2c-e). In summary, in agreement 123 with previous studies 36,37 , our results showed that U373 cells form flat clathrin-coated 124 plaques with a long lifetime exclusively at attached plasma membrane parts. 
126 It has been proposed that the clathrin machinery is involved in recycling of FAs ${ }^{38-40}$. To

127 address whether clathrin-coated plaques might be involved in this process, FAs were

128 labelled in U373 cells by expressing the markers of FAs, zyxin, vinculin, focal adhesion

129 kinase (FAK) or paxillin fused to the fluorescent protein mCherry. Mature FAs and smaller

130 focal complexes did not colocalize with clathrin structures of any size, suggesting that

131 neither CCPs nor clathrin-coated plaques have a physical correlation with FAs (Fig. 1a). To

132 rule out that the lack of colocalization between clathrin-coated plaques and FAs was due to

133 the transient nature of their interaction during the disassembly process, we performed live-

134 cell fluorescence microscopy of migrating U373 cells expressing AP2-eGFP as a marker for

135 clathrin structures and mCherry-zyxin, as a marker for FAs. Interestingly, we observed that

136 during cell migration, clathrin-coated plaques frequently formed at the position where FAs

137 were disassembled (Fig. 1b and e, Supplementary Movie 1). In other words, following

138 disassembly of FAs, clathrin structures are actively assembled at the same locations (Fig.

139 1b). During this switch from FAs to clathrin-coated plaques, the clathrin structures and the

140 FA did not colocalize and were mutually exclusive overtime at a given position (Fig. 1c-d).

141 The formation of clathrin-coated plaques was a process specific to the locations of

142 disassembled of FAs whereas the appearance of clathrin-coated plaques at other locations

143 was a rare event (Fig. 1e). This process was not specific to zyxin as similar mutual

144 exclusions were observed using the FA markers vinculin, FAK, and paxillin (Supplementary

145 Fig. 3). Importantly, this phenomenon was not cell type specific as we could observe a

146 similar switch when using other cell types also known to display clathrin-coated plaques

147 (HT1080 and U20S, Supplementary Fig. 4). These results report a so far undescribed switch

148 from FAs to clathrin-coated plaques and suggest that clathrin-coated plaques have a

149 molecular origin locally imprinted by FAs.

\section{Clathrin-coated plaques are stabilized by integrins}

151 In the context of integrin internalization during FA recycling, the above-described switch

152 from FAs to clathrin-coated plaques could generate an endocytic hot spot for the

153 internalization of integrins during FA disassembly. Most importantly, very recent reports

154 have demonstrated the importance of integrins in stabilizing clathrin-coated plaques ${ }^{33,34}$. By

155 performing immunostaining of endogenous integrins, we could show that integrins $\alpha \mathrm{v} \beta 5$

156 and $\beta 1$ were enriched at clathrin-coated plaques in U373 cells (Fig. 2a-b and Supplementary

157 Fig. 5a, respectively). This suggests that integrins are left behind at the position where FAs 
158 are disassembled and could recruit or stabilize the clathrin machinery to locally promote

159 the formation of long-lived clathrin-coated plaques. To challenge the possible function of 160 integrins in stabilizing clathrin-coated plaques that are formed after FA disassembly, we

161 used the cyclic RGD peptide Cilengitide, which acts as an integrin antagonist and inhibits 162 integrin binding to their ECM ligands ${ }^{41,42}$. We observed that Cilengitide treatment induced 163 the rapid dissociation of clathrin-coated plaques (Fig. 2c-e, Supplementary Movie 2). During 164 Cilengitide treatment, clathrin-coated plaques disassembled by the formation of several 165 smaller transient structures resembling endocytic hot spots (Fig. 2d and Supplementary 166 Movie 2). Together these results indicate that clathrin-coated plaques replace 167 disassembling FAs and are recruited and/or stabilized by integrins.

\section{Extracellular signals drive the formation of clathrin-coated plaques}

169 To address whether a specific extracellular signal created by FAs was left after FA 170 disassembly and was in turn responsible for the integrin-dependent recruitment of clathrin171 coated plaques, we monitored the switch from FAs to clathrin-coated plaques in migrating 172 cells. We found that the location of clathrin-coated plaques is somehow defined by the 173 extracellular environment. Indeed, we observed that clathrin-coated plaques often appear 174 at the same locations when the same migrating cell revisits a position twice (Fig. 3a, first 175 cell at $\mathrm{t}=0$ and first cell at $\mathrm{t}=4.4 \mathrm{~h}$ ). More strikingly, if a different cell migrates over these 176 plaque-forming areas, clathrin-coated plaques are also observed in the new cell at the same 177 positions (Fig. 3a, merge between second cell $t=7.7 \mathrm{~h}$ and first cell $\mathrm{t}=0$ and $\mathrm{t}=4.4 \mathrm{~h}$ ). To 178 unambiguously demonstrate that an extracellular signal was responsible for clathrin-coated 179 plaque formation at the same location, we correlated the location of clathrin-coated plaques 180 on gridded coverslips when cells were seeded sequentially in multiple rounds (Fig. 3b). In 181 this experiment, we seeded first U373 expressing AP2-eGFP on gridded coverslips and 182 imaged clathrin dynamics to identify the locations of clathrin-coated plaques. Following 183 EDTA-mediated removal of the first cells, a second seeding of cells was performed on the 184 same grid and both clathrin dynamics and plaque locations were again recorded. Results 185 show that clathrin-coated plaques formed at the same position in the first and the second 186 round of cell seeding (Fig 3c-d). These results demonstrate that specific extracellular signals 187 are responsible for the local induction of clathrin-coated plaques. Trypsin and KOH-based 188 cleaning of the gridded coverslips between both rounds of cell seeding resulted in a strong 
189 reduction of the number of clathrin-coated plaques forming at the same position (Fig. 3d),

190 suggesting that these extracellular signals are of proteinaceous nature

191 Remodelled ECM represents an extracellular cue that promotes clathrin-coated plaque

\section{2 formation}

193 FAs are supramolecular complexes not only involved in ECM binding, but also responsible

194 for ECM remodelling13,15. One of the important remodelling processes driven by FAs is the

195 enzymatic digestion of the ECM ${ }^{43}$. To directly correlate digestion of ECM and recruitment of

196 clathrin-coated plaques, we employed fluorescently labelled gelatin as a substitute for ECM

197 and monitored live the digestion of the extracellular environment together with the 198 dynamics of clathrin-coated structures. During cell migration, we could observe that FAs 199 located at the leading edge of the cell actively digested the fluorescent gelatin, as can be 200 seen by the appearance of dark non-fluorescent areas (Supplementary movie 3). 201 Interestingly, by monitoring clathrin dynamics on fluorescently labelled gelatin coatings, we 202 unravelled that the locations of long-lived clathrin-coated plaques correlated with the 203 locations of the digested fluorescent gelatin (Fig. 4a-d and Supplementary movie 4, black

204 area). To temporally correlate gelatin digestions by FAs and clathrin-coated plaque 205 formation, U373 cells expressing AP2-eGFP and mCherry-zyxin were seeded on fluorescent 206 gelatin and the dynamics of the switch from FAs to clathrin-coated plaques was observed 207 using live fluorescence confocal microscopy. Analyses revealed that during cell migration, 208 FAs dynamically formed and locally digested the gelatin coat, which was followed by FA 209 disassembly and finally clathrin-coated plaque formation (Fig. 4e-g, Supplementary Movie 210 3). Importantly, in line with the above results showing that two different migrating cells 211 form their clathrin-coated plaques at similar locations (Fig. 3a), our fluorescent gelatin 212 assay reveals that the extracellular cues that lead to clathrin-coated plaques formation 213 correspond to spots of digested ECM (Fig. 4h, Supplementary movie 5). Together, these

214 results strongly suggest a model where FAs digest and modify the ECM creating 215 extracellular domains responsible for the integrin-dependent recruitment of clathrin-coated 216 plaques. 
219 To study the switch from FAs to clathrin-coated plaques and its link to FA disassembly 220 and/or creation of extracellular cues, we exploited a pharmacological approach to induce 221 FA disassembly. We used the ROCK inhibitor Y-27632 previously shown to induce FA 222 disassembly 44,45 . Treatment of U373 cells with Y-27632 induced the disassembly of actin 223 stress fibres and FAs within 20 minutes (Supplementary Fig. 6a-c). By monitoring the 224 dynamics of both the clathrin machinery and of FAs during drug treatment, we could 225 demonstrate that FAs are disassembled and are efficiently replaced by clathrin-coated 226 plaques during the drug-induced FA disassembly (Fig 5a-b, Supplementary Movie 6). This 227 replacement was similar to the process seen during cell migration as FA and clathrin-coated 228 structures were located at the same site but temporally mutually exclusive (Fig. 5b, 229 Supplementary Fig. 6g). Lifetime analyses of the newly formed clathrin-coated structures 230 revealed that they were clathrin-coated plaques (Fig. 5c). Quantification of the efficiency of 231 the drug-induced switch from FAs to clathrin-coated plaques revealed that approximately $23240 \%$ of all FAs were replaced by clathrin-coated plaques (Fig. 5d). Similar to Y-27632233 induced FA disassembly, treatment of cells with the myosin-II inhibitor Blebbistatin also 234 induced FAs to switch to clathrin-coated plaques (Supplementary Fig. 6d-f and h, 235 Supplementary Movie 7). By performing immunostaining of integrins in cells treated with 236 Y-27632, we observed that integrins were enriched at clathrin-coated plaques located at the 237 cell periphery and the overall colocalization of integrins with clathrin structures increased 238 (Fig. 5e-f and Supplementary Fig. 5b). These structures corresponded to the FAs which 239 disassembled during drug treatment, leaving behind integrins which in turn promoted the 240 recruitment of the clathrin coat. By performing the drug-induced FA disassembly assay on 241 labelled gelatin coatings, we could show that clathrin-coated plaques were recruited to 242 former FA sites, which actively digested the ECM (Fig. 5g). Quantification of the frequency of 243 drug-induced switch from FAs to clathrin-coated plaques revealed that most of the FAs 244 which at the time of Y-27632 treatment had actively digested the fluorescent gelatin were 245 replaced by clathrin-coated plaques, in comparison to FAs without visible gelatin digestion 246 (Fig. 5h). These results show that in this experimental set-up, gelatin digestion is an efficient 247 signal for clathrin-coated plaque recruitment after FA disassembly. To further correlate 248 recruitment of clathrin-coated plaques with ECM digestion, we knocked down or 249 overexpressed the matrix metalloproteinase MMP14 in U373 cells (Supplementary Fig. 7). 250 MMP14 is a transmembrane metalloproteinase that plays a key role in digesting the ECM 251 and activating other metalloproteinase and thereby regulates FAs turnover and 
252 subsequently cell migration43,46-50. Upon MMP14 knock-down, we observed that digestion of

253 the fluorescent gelatin was significantly reduced (Fig. 5i-j, Supplementary Fig. 7) and this

254 was associated with a significant reduction of the number of FAs switching to clathrin-

255 coated plaques (Fig. 5k). Interestingly, overexpression of MMP14, which led to global

256 digestion of gelatin coats instead of locally defined digestions as seen for WT cells (Fig. 5i-j,

257 Supplementary Fig. 7), is also associated with a strong reduction of the drug-induced switch

258 (Fig. 5k). Altogether, these results demonstrate that the switch from FAs to clathrin-coated

259 plaques is tightly associated with the FA-mediated digestion of the ECM.

261 Cellular processes, e.g. protein secretion, fibrillogenesis or enzymatic digestion, which occur

262 at cell/extracellular environment contact sites, can rearrange the ultrastructural

263 organization of the ECM113. In this way not only the protein content of the ECM can be

264 changed, but also its physical properties $9,10,51,52$. Therefore, the above observed correlation

265 between the location of clathrin-coated plaques and the site of gelatin digestion could be the

266 result of a change in the topographical organization of the ECM which in turn will induce

267 local plaque formation. To directly investigate whether local topographical cues can induce

268 clathrin-coated plaque formation, we generated 3D-micropatterns suitable for microscopy

269 made of an optically clear glue (Norland Optical Adhesive (NOA)) using a soft lithography

270 approach. These 3D-micropatterns were made from optical gratings and displayed peaks of

$271200 \mathrm{~nm}$ height with a periodicity of $2 \mu \mathrm{m}$ (Supplementary Fig. 8a-c). Analysis of the 3D-

272 micropatterns using atomic force microscopy (AFM) revealed that they precisely

273 reproduced the peak height and periodicity of the optical gratings (Supplementary Fig. 8b-

274 f). U373 cells growing on such 3D-micropatterns align both their FAs and actin stress fibres

275 parallel to the pattern as previously reported with different cell lines (data not shown

276 and ${ }^{53-57}$ ). Interestingly, most clathrin structures were also found aligned parallel to the

277 grating with the same periodicity of $2 \mu \mathrm{m}$ (Fig. 6a-c). Strikingly, analyses of the clathrin

278 structure dynamics of cells seeded on such 3D-micropatterns revealed that the long-lived

279 clathrin-coated plaques only formed on top of the gratings whereas transient CCPs formed

280 independently of the topographical features (Fig. 6d and e). This local induction of clathrin-

281 coated plaque formation by substrates displaying 3D topographical micropatterns again

282 highlights the relation between clathrin-coated plaques and extracellular physical cues. 


\section{Clathrin-coated plaques are promoted by specific CME initiator proteins}

284 Formation of clathrin-coated structures is achieved through multiple adaptor and accessory 285 proteins, which are regulated in space and time to precisely coordinate coat assembly at the 286 ultrastructural level22,23. Most studies that have looked at the functions of different proteins 287 in clathrin coat formation have focused on their function during endocytosis. On the 288 contrary, very little is known about proteins specifically regulating clathrin-coated plaque 289 formation and function ${ }^{27}$. Previously, it was suggested that proteins of the clathrin pioneer 290 module that initiate CME and lead to the recruitment of clathrin to the plasma membrane, 291 e.g. FCH01/2 and Eps15/R, stabilize the clathrin lattice early during coat formation and 292 help to establish the hexagonal organization of flat clathrin structures $34,58-60$. Therefore, we 293 investigated if early clathrin-related proteins of the pioneer module are important for 294 clathrin-coated plaque formation. We found that depletion of Eps15 and Eps15R in U373 295 through shRNA-mediated knock-down (shEPS15/R) resulted in a dramatic reduction in the 296 number of long-lived clathrin-coated plaques (Fig. 7a-b, Supplementary Fig. 9a). In line with 297 their function as a pioneer module, knock-down of Eps15/R was also associated with a 298 reduction in the number of CCP initiation events (Fig. 7a). Importantly, the switch from FAs 299 to clathrin-coated plaques was severely impaired in cell knocked down for Eps15/R, both 300 during cell migration (data not shown) and during Y-27632-induced FA disassembly (Fig. 301 7c-e, Supplementary Fig. 9b). While drug treatment was efficiently inducing disassembly of 302 FAs, only transient CCPs formed occasionally at the sites of disassembled FAs (Fig. 7d-e). Of 303 note, individual knock-down of Eps15 and Eps15R, also resulted in reduction of switch from 304 FAs to clathrin-coated plaques, but to a lesser extent compared to the dual depletion of both 305 Eps15 and Eps15R (Supplementary Fig. 9b). These results show that, in addition to their 306 role in the initiation of CCP formation as part of their pioneer module function ${ }^{60-62}$, Eps15/R 307 also play a role in regulating plaque formation and FA switch to clathrin-coated plaques.

308 Cell migration is influenced by substrate topography through clathrin-coated plaque 309 formation

310 Topographical cues of the ECM have been shown to influence the direction of cell 311 migration ${ }^{10,57,63}$. This phenotype called contact guidance plays an important role during 312 development ${ }^{64-66}$ as a well as tumour metastasis 67,68 . Our findings that clathrin-coated 313 plaques can form after FA disassembly and the fact that this recruitment appears to be 
314 linked to the creation of extracellular cues in the ECM, led us to hypothesize that clathrin-

315 coated plaques might participate in contact guidance-mediated directionality of cell

316 migration by recognizing the extracellular cues generated by FAs in the ECM. To study the

317 influence of clathrin-coated plaques on the directionality of cell migration, we followed

318 U373 cells on 3D-micropatterns as well as on flat substrates made of the same material and

319 we characterized the contact guidance-mediated directionality of cell migration. As

320 described above, WT U373 displayed clathrin-coated plaques aligned with the 3D-

321 micropatterns (Fig. 8a) and we found that migration of these cells was directed parallel to

322 the grating following the grid lines (Fig. 8c, e (right panel) and g). In comparison, WT U373

323 cells display random cell migration on flat surfaces made of the same optically clear glue

324 (Fig. 8e and g). As expected, Eps15/R knock-down cells seeded on the 3D-micropattern

325 failed to display clathrin-coated plaques (Fig. 8b). Importantly, cell migration analysis of

326 Eps15/R knock-down cells on 3D-micropatterns revealed that cells depleted of clathrin-

327 coated plaques do not show directional migration along the grating orientation (Fig. 8b, d, $f$

328 and g). Altogether, these results indicate that clathrin-coated plaques participate in contact

329 guidance-mediated directionality of cell migration.

\section{Discussion}

331 Here, we describe how modifications of the ECM can lead to recruitment of clathrin-coated 332 plaques and correlate their spatio-temporal formation with the regulation of cell migration. 333 We demonstrate that clathrin-coated plaque formation is associated with the modification 334 of the extracellular environment by FAs. Precisely, by combining live monitoring of ECM 335 digestion/modifications and dynamics of clathrin-coated plaque formation, we show that, 336 during cell migration, metalloproteinases located in FAs, digest the ECM creating specific 337 extracellular cues which in turn drive the formation of flat clathrin arrays. Localization of 338 clathrin-coated plaques and FAs at these specific sites reveal that these structures are 339 temporally mutually exclusive. We name this process "FA switch to clathrin-coated 340 plaques". By exploiting 3D-micropatterns, we show that these extracellular signals

341 represent topographical cues, which are recognized by the clathrin machinery inducing the

342 local formation of plaques. We identify Eps15 and Eps15R as key regulators of plaque 343 formation. Finally, we show that by recognizing the extracellular topographical cues, 344 clathrin-coated plaques regulate the directionality during cell migration. 
345 The presence of clathrin-coated plaques at the ventral plasma membrane has been long

346 known $^{36}$. Recently, although their origin and function remained enigmatic, several studies

347 have reported that the presence of clathrin-coated plaques at the plasma membrane was

348 dependent on integrins ${ }^{33,34}$. These findings described that not only are integrins enriched at

349 clathrin-coated plaques, but also describes that they are mandatory to stabilize flat clathrin

350 arrays $27,33,34$. This is consistent with our findings that interference with integrin binding to

351 the ECM proteins impacts clathrin-coated plaque stability and induces their disassembly.

352 The cyclic RGD peptide Cilengitide was recently shown to be a competitive antagonist which

353 exerts its activity only on integrins which are not bound to ECM RGD motifs ${ }^{69}$. As such the

354 observation that Cilengitide can induce plaque disassembly (this work and ${ }^{33}$ ) suggests that

355 although plaques are stable structures at the plasma membrane, their integrin-dependent

356 binding to the ECM is highly dynamic. Importantly, our work also suggests that the presence

357 of integrin clusters is necessary, but not sufficient for clathrin-coated plaques. A great

358 number of integrin clusters can be found at the plasma membrane, but not associated with

359 the formation of clathrin-coated plaques (Fig. 2, Supplementary Fig. 5). Recent work by

360 Baschieri et al. highlighted the connection of clathrin-coated plaque formation and physical

361 properties of the substrate suggesting a mechanosensing function of plaques. They showed

362 that stiff ECM substrates favour the formation of long-lived clathrin arrays by preventing

363 receptors (e.g. integrins) from being internalized ${ }^{33}$. In light of our findings, FAs-mediated

364 remodelling might change the physical properties of the ECM leading to the induction of

365 clathrin-coated plaques. We could show that topographical cues of the substrate (i.e. gelatin

366 digestions and 3D-micropatterns) are potent signals for plaque formation in an integrin-

367 dependent manner. Furthermore, ECM remodelling can change the mechanical properties

368 of the substrate that could be sensed by clathrin-coated plaques ${ }^{70-72}$.

369 The presence of extracellular 3D nanostructures has been previously described to be able to 370 induce the formation of clathrin-coated structures ${ }^{18-21}$. Endocytically active CCPs are found

371 to be induced both by artificial nanostructures 18,20 and by virus particles which impose local

372 membrane curvature 21 . Recruitment and/or induction of clathrin lattice assembly has

373 previously been proposed to be linked with the specific recruitment of membrane curvature 374 sensing proteins like N-BAR proteins ${ }^{18}$. Extracellular collagen fibres have also been

375 reported to induce the formation of a unique clathrin coat called tubular clathrin/AP2

376 lattices (TCALs) ${ }^{19}$. Although TCALs seem to have some similarities to clathrin-coated 
377 plaques, it is not clear if they describe the same phenomenon in a different context. Both of 378 these structures contain integrin receptors and form at locally defined ECM structures. One 379 important difference between TCALs and clathrin-coated plaques emanating from a FA 380 switch is that TCALs only form in a complex 3D environment and are of a more transient 381 nature. On the contrary, clathrin-coated plaques are stable for hours and form in classical 382 2D cell culture but are also induced by extracellular 3D features.

383 We have identified Eps15 and Eps15R as key players for clathrin-coated plaque formation 384 and for the switch from FAs clathrin-coated plaques. Additional to their function in 385 initiation of CCPs as part of the pioneer module ${ }^{60-62}$, a recent study describes that together 386 with the clathrin adaptor Numb, Eps15/R contribute to integrin $\beta 5$ localization in flat 387 clathrin lattices ${ }^{34}$. By binding to the integrin $\beta 5$ cytoplasmic domain, the Numb-Eps15/R

388 complex links integrins to the clathrin coat and helps to establish integrin-containing 389 clathrin-coated plaques. This mechanism could explain why cells depleted from Eps15/R 390 fail to form clathrin-coated plaques after FA disassembly.

391 During migration, we show that cells actively remodel their surrounding and generate 392 topographical cues. Importantly, formation of these cues correlates with the integrin393 dependent formation of clathrin-coated plaques after FA disassembly. Additionally, using 394 our 3D-micropatterns, we could demonstrate that topographical cues can directly promote 395 the formation of clathrin-coated plaques. As such, it tempting to propose that during cell 396 migration, the FA-mediated topographical cues represent the signal leading to plaque 397 formation. This model is supported by the fact that removing these topographical cues 398 indeed leads to a loss of clathrin-coated plaque formation. Specifically, we could show that 399 when cells are either depleted of or overexpressing the matrix metalloproteinase MMP14, 400 the ECM is not digested or fully digested, respectively (Fig. 5i-j). On these surfaces which are 401 lacking topographical cues (either because there is a lack of local digestion or because there 402 is a complete digestion of the entire surface), switch from FAs to clathrin-coated plaques 403 was severely impaired (Fig. 5k). Similarly, in our cell seeding experiments (Fig. 3b-c), when 404 the ECM was removed by treatment of the surfaces using either $\mathrm{KOH}$ or trypsin, a loss of 405 spatial colocalization of plaque between the two rounds of seeded cells was observed (Fig. 406 3d). As such we propose that FAs generate extracellular guidance patterns which will be 
407 recognized by clathrin-coated plaques ultimately influencing cell migration and establishing

408 directionality along these topographical cues.

409 The exact mechanisms by which plaques can influence cell migration remains to be

410 determined. Several studies report clustering and recruitment of transmembrane receptors

411 and signalling molecules at clathrin-coated plaques $29,30,33$. Their stable nature would provide

412 the cells with a long-lasting signalling platform which could influence fundamental

413 processes regulating cell migration. However, in such a model, it is hard to explain how the

414 presence of plaques can influence migration directionality. In the case of FA assembly and

415 turnover, it has been proposed that internalizing integrin at the rear and their transport to

416 the front of migrating cells might contribute to the establishment of directionality 73,74 . As

417 such, it is possible that a similar gradient of integrin interaction with and/or integrin

418 recycling by clathrin-coated plaques participates in directional cellular migration.

419 The regulation of clathrin-coated plaques and the formation of cell type-specific clathrin

420 structures might serve as topography sensing protein complexes and influence cellular

421 behaviour due to ECM features. In the context of a population of cells, our results support a

422 model where a leader cell will create extracellular cues through FA switch to clathrin-

423 coated plaques, which will then be recognized by the following cells influencing its

424 migration. The best characterized mechanisms leading to directed cell migration is

425 chemotaxis where cells have the ability of sensing external gradient of chemotactic

426 factors 55 . In the complex environment of tissue in a whole organism, chemotactic gradients

427 are further shaped by ECM (diffusion and immobilization) and neighbouring cells (secretion

428 and sequestering) ${ }^{75}$. Importantly, cells not only respond to chemical gradients but also have

429 been described to respond to mechanical gradients within the extracellular space

430 (durotaxis) 75 . As such it is possible that clathrin-coated plaque might participate in

431 regulating a unique type of collective migration, where cell-to-cell contact is dispensable

432 and where the collective behaviour comes from sensing extracellular topographical cues left

433 by the preceding cells. The formation of clathrin-coated plaques and its impact on guiding

434 cell migration during contact guidance as well as collective cell migration might be a highly

435 important process in development as well as tumour migration. 


\section{Materials and Methods}

\section{Cell culture}

438 U373, HT1080, and U2OS cells were maintained in DMEM (Gibco, 41965-039) and Hek293T

439 cells for Lentivirus production were maintained in IMDM (Gibco, 21980-032) both

440 supplemented with 10\% FBS (Biochrom, S0615) and Penicillin/Streptomycin (Gibco,

44115140 ) at $37^{\circ} \mathrm{C}$ and $5 \% \mathrm{CO}_{2}$. Sf9 were maintained in Sf-900 III SFM (Gibco, 12658019) at $442 \quad 27^{\circ} \mathrm{C}$.

\section{Plasmids, shRNA and antibodies}

444 Plasmids for mammalian expression of mCherry-FAK (55044), mCherry-paxillin (55114), 445 and mCherry-vinculin (55160) were purchased from Addgene. Mammalian expression 446 plasmid for mCherry-zyxin used for the cloning was a gift from Benjamin Geiger. The 447 gateway vector pDONR 221 (12536017) and BacMam pCMV-DEST (A24223) were 448 purchased from Invitrogen and were used for cloning of BacMam pCMV mCherry-zyxin to 449 generate recombinant bacmid DNA for BacMam production. Mammalian expression 450 plasmid containing the rat AP2 subunit sigma2 C-terminally fused to eGFP 76 was used for 451 the generation of stable cell lines. The lentiviral backbones pLK0.1 puro was a gift from Dr. 452 Björn Tews and pLKO.1 blast (26655) was purchased from Addgene and were used for 453 cloning of shRNA expression vectors for lentivirus production. The gateway entry vector 454 pENTR MMP14 was obtained from the DKFZ Vector and Clone Repository and the gateway 455 destination vector pWPI puro (Invitrogen) was used for cloning of MMP14 expression 456 vector for lentivirus production. The VSV-G envelope expressing plasmid pMDG.2 (12259) 457 and the lentiviral packaging plasmid psPAX (12260) were purchased from Addgene were 458 used for lentivirus production.

459 The target sequences shscrambled (shscr) (5' CAACAAGATGAAGAGCACCAA 3'), shMMP14 460 (5' CATTGCATCTTCCCTAGATAG 3'), shEPS15 (5' CCCAGAATGGATTGGAAGTTT 3'), and 461 shEPS15R (5' GAAGTTACCTTGAGCAATC 3') were used for the shRNA-mediated protein 462 knock down. 
463 Monoclonal antibody against $\alpha v \beta 5$ integrin (ab177004; used 1:100 for IF), MMP14 464 (ab51074; used 1:100 for IF and 1:5,000 for WB) and Eps15R (EP1146Y; used 1:5,000 for

465 WB) were purchased from Abcam. Monoclonal antibody against $\beta$ actin (A5441; used 466 1:5,000 for WB) and vinculin (V9131; used 1:800 for IF) and polyclonal antibody against 467 Eps15 (HPA008451; used 1:5,000 for WB) were purchased from Sigma. Monoclonal 468 antibody against $\beta 1$ integrin (552828; used 1:1,000 for IF) was purchased from BD. The 469 secondary antibodies anti-mouse IgG HRP (NA931; used 1:5,000 for WB) and anti-rabbit 470 IgG HRP (NA934; used 1:5,000 for WB) were purchased from GE Healthcare. The secondary 471 antibodies anti-mouse Alexa Fluor 568 (A-11004), anti-mouse Alexa Fluor 647 (A-21235), 472 and anti-rat Alexa Fluor 647 (A-21247) were purchased from Invitrogen.

\section{Western blot (WB)}

474 For Western blot (WB) analysis, protein samples from cell lysates were produced. Cells 475 were detached and pelleted ( $300 \mathrm{xg}, 3$ minutes). After one time washing with PBS, cells 476 were pelleted again and the cell pellet was lysed with appropriate volume of NP-40 buffer 477 (50 mM Tris $\mathrm{pH}$ 8.0, $150 \mathrm{mM}$ sodium chloride, 1\% (v/v) NP-40, protease inhibitor (Roche, 478 118735800001)) for 30 minutes on ice. The cell lysate was spun down for 20 minutes at $47912,000 \mathrm{x} g$ and the supernatant was transferred to a fresh tube.

480 To measure the protein concentration of protein samples, DC (detergent compatible) 481 Protein Assay Kit II (BioRad, \#5000112) was used and compared to a bovine serum 482 albumin (BSA) protein standard. To compare amounts of proteins of interest in different 483 samples, the concentration of the samples was adjusted by diluting higher concentrated 484 samples with NP-40 buffer.

485 Sodium dodecyl sulphate polyacrylamide gel electrophoresis (SDS-PAGE) gels were 486 prepared with appropriate concentrations of the separating gel. Protein samples were 487 mixed with 4x Laemmli buffer (0.2 M Tris-HCl pH 6.8, 0.05 M EDTA, 40\% (v/v) glycerol, 8\% $488(\mathrm{w} / \mathrm{v})$ SDS, 4\% (v/v) $\beta$-mercaptoethanol, 0.03\% (w/v) bromophenol blue), heated for 48910 minutes at $95^{\circ} \mathrm{C}$ and spun down for 1 minute at $12,000 \mathrm{x}$ g before loading. As molecular 490 weight marker $10 \mu \mathrm{l}$ Precision Plus Protein Dual Color Standards (BioRad, 1610374) was 491 separated together with the protein samples in SDS-Tris-Glycine buffer (25 mM Tris-base, $492200 \mathrm{mM}$ Glycine, 1\% (w/v) SDS). SDS-PAGE was first performed at $80 \mathrm{~V}$ until samples 
493 moved through the stacking gel and then turn up to $100 \mathrm{~V}$ until loading dye run out of the

494 gel. Proteins were transferred to a nitrocellulose blotting membrane $(0.45 \mu \mathrm{m}$; GE 495 Healthcare, \#10600003) by wet blot using transfer buffer (20 mM Tri-base, $160 \mathrm{mM}$

496 Glycine, 20\% methanol) at $100 \mathrm{~V}$ for 1 hour. Then membranes were blocked with $5 \%$ milk 497 in TBST (50 mM Tris-HCl pH 7.5, $150 \mathrm{mM} \mathrm{NaCl}, 0.1 \%$ Tween) for 1 hour at room 498 temperature. Incubation with primary antibody diluted in TBST with 5\% milk was 499 performed overnight at $4^{\circ} \mathrm{C}$. After four washes for 5 minutes with TBST, membranes were 500 incubated with secondary antibody diluted in TBST with 5\% milk for 1 hour at room 501 temperature. The membranes were washed four times for 5 minutes with TBST and 502 developed with ECL Western Blotting Detection Reagents (GE Healthcare, RPN2106) or 503 Western Bright Chemilumineszenz Substrate Sirius (Biozym, 541021) using high 504 performance chemiluminescence films (GE Healthcare, \#28906837).

\section{Cloning of short hairpin RNA (shRNA) encoding vector for lentivirus production}

506 Forward and reverse oligonucleotides containing short hairpin RNA (shRNA) sequence 507 were designed according to RNA interference (RNAi) consortium (TRC) 508 (https://www.broadinstitute.org/scientific-community/science/projects/rnai-

509 consortium/rnai-consortium) and ordered from Eurofins. Complementary oligonucleotides 510 were designed to generate 5' and 3' overhangs after annealing complementary to AgeI and 511 EcoRI restriction sites, respectively, for direct ligation into linearized vectors. While the 512 EcoRI restriction site was kept intact by the oligonucleotide insertion, the AgeI restriction 513 site was destroyed, which was used for test digestions as described later. Oligonucleotides 514 were diluted in $\mathrm{H}_{2} \mathrm{O}$ at a concentration of $1 \mu \mathrm{g} / \mu \mathrm{l} .2 .5 \mu \mathrm{l}$ of forward and reverse 515 oligonucleotides were mixed with $5 \mu \mathrm{l}$ NEB Buffer 2 (NEB, B7002S) and $40 \mu \mathrm{l} \mathrm{H}_{2} \mathrm{O}$. The 516 oligonucleotide mix was heated at $95^{\circ} \mathrm{C}$ for 5 minutes and slowly cooled down to room 517 temperature for annealing of both strands. For the ligation reaction, $150 \mathrm{ng}$ of pLKO.1 518 backbone, which has been sequentially digested with EcoRI (R0101S) and AgeI-HF 519 (R3552S) purchased from NEB, and $2 \mu \mathrm{l}$ annealed oligonucleotides were used. A $20 \mu \mathrm{l}$ 520 ligation reaction was set up using T4 Ligase (NEB, M0202S) and incubated for 1 hour at 521 room temperature. The whole ligation mix was used for transformation into DH5 $\alpha$ 522 (Invitrogen, 18265017). 
523 Colonies were tested by digestion with AgeI-HF and BamHI (NEB, R0136S). Insertion of

524 oligonucleotides containing shRNA led to the loss of AgeI restriction site. Therefore

525 plasmids with successful insertion showed only one linearized vector band whereas re-

526 ligated plasmids were recognized by a double band. Plasmids were further controlled by

527 sequencing with U6 primer.

\section{Lentivirus production}

529 For lentivirus production, Hek293T cells were cultured in $10 \mathrm{~cm}$ dishes until 80\% 530 confluence. The cells were transfected with pMDG.2, psPAX, and the shRNA encoding 531 pLKO.1 vector or the pWPI vector containing the gene of interest using PEI. $50 \mu \mathrm{l}$ of PEI $532(1 \mathrm{mg} / \mathrm{ml})$ was diluted in $200 \mu \mathrm{l}$ Opti-MEM (Gibco, 31985062) and mixed well. $4 \mu \mathrm{g}$ pMDG.2, $5334 \mu \mathrm{g}$ psPAX, and $8 \mu \mathrm{g}$ pLKO.1 or pWPI were mixed with Opti-MEM to a final volume of $250 \mu \mathrm{l}$ 534 and mixed well. After 5 minutes, both solutions were mixed, vortexed and incubated for 53520 minutes at room temperature. Then the transfection mix was added dropwise to cells.

536 Culture medium was exchanged the next day. After another two days, the supernatant was 537 harvested, centrifuged (10 minutes, 4,000 x g) and filtered through a syringe filter (Millex538 HA, $0.45 \mu \mathrm{m}$, Millipore, SLHA033SS). For short time storage, the lentivirus containing 539 supernatant was kept at $4^{\circ} \mathrm{C}$; for long time storage, the supernatant was aliquoted and 540 stored at $-80^{\circ} \mathrm{C}$.

\section{Gateway Cloning}

542 The Gateway Technology (Invitrogen) was used for generating entry and expression 543 plasmids. This highly efficient cloning system is based on site-specific recombination 544 reactions using recombination sequences (att-sites) and enzymes, which catalyse 545 recombination reactions (Clonases). This system uses a set of donor and destination vectors 546 to quickly move DNA sequences between multiple vectors. Donor and destination vectors 547 contain a cassette flanked by att-sites, which hold the $c c d B$ gene for negative selection after 548 recombination reaction and a chloramphenicol resistance gene $\left(\mathrm{CM}^{\mathrm{R}}\right)$ for counterselection

549 during propagation. Therefore, donor and destination vectors need to be propagated in 550 E.coli strains resistant to $\mathrm{CcdB}$ effects. This cassette is removed by the recombination 551 reactions and replaced by the gene of interest. More details on the system and the 552 procedure can be found in the manufacturer's manual. 
553 To generate entry vectors Gateway BP Clonase II Enzyme Mix (Invitrogen, 11789) was used.

554 In brief, BP-recombination reactions between PCR products containing attB-sites and

555 pDONR 221 containing attP-sites were set up by mixing 150 ng of PCR product with $150 \mathrm{ng}$

556 of pDONR 221. TE buffer (10 mM Tris-HCl pH 7.4, 1 mM EDTA) was added to reach a final

557 volume of $8 \mu \mathrm{l}$. Then, $2 \mu \mathrm{l}$ of BP Clonase II Enzyme Mix were added and incubated for 1-

5583 hours at $25^{\circ} \mathrm{C} .1 \mu \mathrm{l}$ of Proteinase K (Invitrogen, AM2548) was added to the reaction mix

559 and incubated for 10 minutes at $37^{\circ} \mathrm{C}$. The reaction mix was transformed into DH5 $\alpha$.

560 Plasmid DNA was isolated and sequenced with M13 forward and reverse primers.

561 To generate expression vectors Gateway LR Clonase II Enzyme Mix (Invitrogen, 11791) was

562 used. In brief, LR-recombination reactions between Gateway entry vectors containing attL-

563 sites and Gateway destination plasmids containing attR-sites were set up by mixing $150 \mathrm{ng}$

564 of the entry vector with $150 \mathrm{ng}$ of the destination vector. TE buffer was added to reach a

565 final volume of $8 \mu \mathrm{l}$. Then, $2 \mu \mathrm{l}$ of LR Clonase II Enzyme Mix were added and incubated for 1-

5663 hours at $25^{\circ} \mathrm{C} .1 \mu \mathrm{l}$ of Proteinase $\mathrm{K}$ was added to the reaction mix and incubated for

56710 minutes at $37^{\circ} \mathrm{C}$. The reaction mix was transformed into DH5 $\alpha$.

568 BacMam production

569 ViraPower BacMam Expression System (Life Technologies, A34227) was used to design and

570 produce BacMam mCherry-zyxin for transduction of mammalian cell lines following the

571 user guide.

572 In brief, the Gateway cloning system was used to generate the Gateway expression plasmid

573 BacMam pCMV mCherry-zyxin. First, a Gateway entry plasmid containing the mCherry-

574 zyxin sequence (pENTR mCherry-zyxin) was generated. The sequence was amplified form

575 the mammalian expression plasmid mCherry-zyxin using the primers mCherry-zyxin

576 forward (5')

577 GGGGACAAGTTTGTACAAAAAAGCAGGCTCAACCATGGTGAGCAAGGGCGAGGAGGATA 3’) and

578 reverse (5' GGGGACCACTTTGTACAAGAAAGCTGGGTCTTACGTCTGGGCTCTAGCAGTGTG 3')

579 to flank the sequence with attB-sites by PCR. The PCR product was used to generate the

580 pENTR mCherry-zyxin by BP-recombination reaction. pENTR mCherry-zyxin was then used

581 to generate the BacMAM pCMV-DEST mCherry-zyxin expression plasmid by LR-

582 recombination reaction. 
583 For transposition of the mCherry-zyxin gene into the bacmid, DH10Bac (Invitrogen, 584 10361012) were transformed and incubated for two days at $37^{\circ} \mathrm{C}$. Using a blue/white 585 screening, positive colonies were selected which contained the recombinant bacmid. The 586 bacmid DNA was isolated following the protocol described in the user guide.

587 To produce BacMam mCherry-zyxin, insect cells were transfected with the bacmid 588 mCherry-zyxin using Cellfectin II reagent (Invitrogen, 10362100) to generate a P1 viral 589 stock. This P1 baculoviral stock was used to amplify BacMam mCherry-zyxin by infection of 590 insect cells. This time, the infected insect cells were culture in a spinner flask to produce a 591 high viral titre. Two rounds of amplification were performed to produce a P2 and a P3 592 baculoviral stock. 3\% FBS was added to the P3 baculoviral stock of BacMam mCherry-zyxin 593 and stored in aliquots at $-80^{\circ} \mathrm{C}$.

\section{$594 \quad$ Transfection and viral transduction}

595 Transfection of cells was done using Lipofectamine 2000 (Invitrogene, 11668027) if not 596 stated otherwise. Cells were plated in 6-well plates one day before transfection. The next 597 day, cells were transfected at 70-80\% confluence. $2 \mu \mathrm{g}$ DNA and 4-8 $\mu$ l Lipofectamine 2000 598 were separately mixed with $100 \mu \mathrm{l}$ OptiMEM. The two solutions were mixed together. After 599 incubation for 20 minutes at room temperature, the transfection mix was added drop-wise 600 onto the cells. For generation of stable cell lines, the growth medium was exchanged for 601 fresh growth medium after 8 hours. The cells were put under selection two days after 602 transfection, selected for 2 weeks and sorted using FACS. For live-cell imaging of cells 603 transiently expressing fluorescently tagged FA proteins, the growth medium was exchange 604 for fresh growth medium after 8 hours. The transfected cells were seeded 24 hours after 605 transfection and imaged 6-8 hours after seeding.

606 For transient expression of mCherry-zyxin, cells were transduced with BacMam mCherry607 zyxin. Cells were seeded with BacMam mCherry-zyxin in appropriate dishes and were used 608 for experiments 1 day after seeding. $3.5 \mu \mathrm{l}$ of the P3 stock was used per 10,000 cells. For 609 lentiviral transduction for generation of stable cell lines, 10,000 cells per well were seeded 610 into 6-well plates together with $500 \mu \mathrm{l}$ of lentivirus containing supernatant. After 2 to 6113 days, growth medium was exchange to selection medium with appropriate antibiotics. 612 Cells were sub-cultured in selection medium for 2 weeks and then used for experiments. 


\section{Widefield epifluorescence microscopy}

614 If not stated otherwise, widefield epifluorescence microscopy was performed with an 615 inverted Ti microscope (Nikon) with a 20x (0.75 numerical aperture, Plan Apo $\lambda$, Nikon) dry

616 objective or a 40x (1.3 numerical aperture, Plan Fluor, Nikon) oil immersion objective and a 617 digital camera (DS-Qi1MC, Nikon) equipped with LED (Lumencor-Sola) as light source.

\section{Spinning disc confocal microscopy}

619 Confocal live-cell imaging was performed with an inverted spinning disc confocal 620 microscope (Nikon, PerkinElmer) with a 60x (1.42 numerical aperture, Apo TIRF, Nikon) or 621 100x (1.4 numerical aperture, Plan Apo VC, Nikon) oil immersion objective and a CMOS 622 camera (Hamamatsu Ocra Flash 4) or an EMCCD camera (Hamamatsu C9100-23B). An 623 environment control chamber was attached to the microscope to maintain cells at $37^{\circ} \mathrm{C}$ and $6245 \% \mathrm{CO}_{2}$. If not stated otherwise, live-cell imaging was performed in DMEM without phenol 625 red (Gibco, 21063-029) containing 10\% FBS.

\section{Total internal reflection fluorescence (TIRF) microscopy}

627 TIRF microscopy was performed with an inverted Ti microscope (Nikon) with objective 628 TIRF illumination, with a 60x (1.49 numerical aperture, Apo TIRF, Nikon) oil immersion 629 objective and an EMCCD camera (Andor iXon Ultra DU-897U).

\section{Quantification of FA replacement by clathrin-coated plaques during cell migration}

631 To quantify the specific formation of clathrin-coated plaques at the position of former FAs 632 during cell migration, live-cell confocal spinning disc was performed on U373 cells stably 633 expressing AP2-eGFP and transiently expressing mCherry-zyxin. The position of all FAs at a

634 given time point was used to check for replacement by clathrin-coated plaques within the 635 following 2 hours. Only FAs that were at a position still covered by the cell after this time 636 were analysed. Each FA was categorized into either replaced by clathrin-coated plaques or 637 not. The formation of long-lived ( $>10 \mathrm{~min}$ ) clathrin-coated plaques was manually verified. As 638 a control, random positions with the same size, which did not show any zyxin signal were 639 analysed in the same way. 
641 Cells growing on glass coverslips (\#1.5, diameter $12 \mathrm{~mm}$ purchased from Thermo Scientific 642 or $24 \mathrm{~mm}$ purchased from Marienfeld) or glassbottom dishes (ibidi, 80827) for TIRF were 643 washed once with PBS and fixed with PFA, formaldehyde, or methanol (only for $\alpha \mathrm{v} \beta 5$ 644 integrin staining). Precisely, cells were incubated either with $2 \%$ PFA or $4 \%$ formaldehyde 645 in PBS for 20 minutes at room temperature or overnight at $4^{\circ} \mathrm{C}$ or with ice cooled methanol 646 for 10 minutes at $-20^{\circ} \mathrm{C}$. All following steps were performed at room temperature. After 647 three washes with PBS, cells were permeabilized with either 0.5\% TritonX in PBS for 64815 minutes or $0.05 \%$ saponin (only for $\alpha v \beta 5$ integrin staining) in PBS for 10 minutes 649 followed by a blocking step with 1\% BSA in PBS for 1 hour. Samples were incubated with 650 appropriate primary antibody dilution in 1\% BSA in PBS for 1 hour. After three washes with 651 PBS, samples were stained with appropriate secondary antibody and/or Phalloidin labelled 652 with Alexa Fluor 647 (Invitrogen, A22287) for 45 minutes followed by four washes with 653 PBS. For normal microscopy, samples were washed with $\mathrm{H}_{2} \mathrm{O}$ and mounted with ProLong 654 Gold antifade reagent with DAPI (Invitrogen, P36931). For TIRF microscopy, samples were 655 fixed with 4\% formaldehyde solution for 20 minutes, washed three times with PBS and kept 656 in PBS.

\section{Inhibitor-induced disassembly of FAs}

658 For inhibitor-induced disassembly of FAs either the ROCK inhibitor Y-37632 659 dihydrochloride (Enzo, ALX-270-333) or Blebbistatin (Sigma, B0560) were used with a final 660 concentration of $10 \mu \mathrm{M}$ or $20 \mu \mathrm{M}$, respectively. The inhibitors were diluted in medium with $66110 \%$ FBS. To check the effect of these inhibitors on FAs and the actin cytoskeleton, cells 662 were incubated with pre-warmed inhibitor-containing medium and fixed with 663 formaldehyde. FAs and the actin cytoskeleton were stained using an antibody against 664 vinculin and phalloidin, respectively. Widefield epifluorescence microscopy was used to 665 quantify the presence of FAs and actin stress fibres in each cell. To analyse the lifetime of 666 clathrin structures forming after inhibitor-induced FA disassembly, each FAs visible before 667 the application of the drug was followed over 30 minutes of treatment and categorized 668 according to the formation of clathrin structures at its position after FA disassembly. The 669 lifetime of clathrin structures appearing during that time were analysed manually and 670 differentiated between transient pits $(<10 \mathrm{~min})$ and long-lived plaques $(>10 \mathrm{~min})$. 
671 To quantify the inhibitor-induced switch from FAs to clathrin-coated plaques, cells 672 expressing AP2-eGFP as well as mCherry-zyxin were used. 10 cells per sample were imaged 673 before and after treatment with pre-warmed inhibitor-containing medium. To correct for

674 lateral shift (pixels shifts) of the samples during the exchange of medium, the images before 675 and after the inhibitor treatment were re-aligned using clathrin-coated plaques or other 676 immotile fluorescent spots as reference landmarks. An automated image analysis workflow 677 by KNIME (https://www.knime.com) was used to perform object-based colocalization 678 analysis of FAs and clathrin structures to quantify the switch. A binary mask for the 679 mCherry-zyxin signal before and the AP2-eGFP signal after the inhibitor treatment was used 680 to check for overlapping objects. Only FAs that were at a position still covered by the cell 681 after the treatment were analysed. As a control for thresholding, the number of overlapping 682 objects of the mCherry-zyxin and the AP2-eGFP signal before the inhibitor treatment was 683 determined and subtracted for normalization.

\section{Inhibition of integrins}

685 For inhibition of integrins, the cyclic pentapeptide Cilengitide (Selleckchom, S7077) was 686 used. The inhibitor was diluted in medium containing 10\% FBS at a final concentration of $68710 \mu \mathrm{M}$. Live-cell spinning disc confocal microscopy of CME dynamics of the same cell was 688 performed before and after drug treatment with a frame rate of 3 seconds to follow 689 clathrin-coated plaque disassembly. To calculate the numbers of clathrin-coated plaques, 690 CME was tracked during 30 minutes of Cilengitide treatment. The movie was separated into 6915 minute long sections and the number of clathrin structures that stayed for the whole 6925 minutes was calculated for each section. This numbers were normalized to the first 693 section directly after applying the drug.

\section{Quantification of colocalization between integrins and AP2}

695 TIRF microscopy images of cells expressing AP2-eGFP and immunostained for $\alpha \mathrm{v} \beta 5$ were 696 analysed using the ImageJ/Fiji plugin Coloc2. As indicated, the Pearson correlation 697 coefficient was calculated from background corrected images either for the whole cell or 698 areas covering FAs or clathrin-coated plaques with the same size. 
ECM degradation assays were performed as previously described ${ }^{77}$. To prepare labelled gelatin, a solution of $0.2 \%$ gelatin (from porcine skin; sigma (G-2500)) in PBS was prepared.

702 To dissolve the gelatin, the solution was heated up to $37^{\circ} \mathrm{C}$ for 30 minutes. For sterilization, 703 the solution was filtered using a syringe filter membrane (Millex-GS, $0.22 \mu \mathrm{m}$, Millipore, 704 SLGS033SB). To label the gelatin with Alexa Fluor 647, $500 \mu \mathrm{l}$ of $0.2 \%$ gelatin solution was 705 preheated to $37^{\circ} \mathrm{C}$ for 30 minutes. $5 \mu$ of Alexa Fluor 647 NHS Ester $(10 \mathrm{mg} / \mathrm{ml}$, Invitrogen, 706 A37573) was added and incubated for 1 hour at room temperature protected from light. To 707 remove the free dye, the labelling mix was dialyzed with a Slide-A-Lyzer MINI Dialysis 708 Device (20 kMWCO, $0.5 \mathrm{ml}$; Thermo Scientific (88402)) against PBS for 2 hours at room 709 temperature. After changing the PBS, the mix was dialyzed overnight at $4^{\circ} \mathrm{C}$. During dialysis, 710 the labelled gelatin was protected from light.

711 To coat coverslips with labelled gelatin, washed coverslips were first coated with poly-L712 lysine (PLL Sigma, P8920), using a $50 \mu \mathrm{g} / \mathrm{ml}$ solution in PBS for 20 minutes at room 713 temperature. The coverslips were washed three times with PBS. Then the PLL coat was 714 fixed with 0.5\% glutaraldehyde (Sigma, G5882) in PBS for 15 minutes at room temperature. 715 The coverslips were washed three times with PBS. Mixture of $0.2 \%$ labelled and unlabelled 716 gelatin in a ratio of $8: 1$ was preheated to $37^{\circ} \mathrm{C}$ for 30 minutes. Coverslips were inverted on a 717 drop of gelatin mix ( $80 \mu \mathrm{l}$ for $12 \mathrm{~mm}$ diameter coverslips; $120 \mu \mathrm{l}$ for $25 \mathrm{~mm}$ diameter 718 coverslips) and incubated for 10 minutes at room temperature. Coverslips were washed 719 three times with PBS. The coated coverslips were directly used for experiments.

720 To seed cells on coverslips coated with labelled gelatin, detached cells were resuspended in 721 DMEM containing 10\% FBS. The cells were pelleted by centrifugation (300 x g, 3 minutes) 722 and washed with PBS to remove residual trypsin. Cells were pelleted again and 723 resuspended in DMEM containing 10\% FBS and plated on coverslips with fluorescent 724 gelatin matrix (20,000 cells for $12 \mathrm{~mm}$ diameter coverslips; 80,000 cells for $25 \mathrm{~mm}$ 725 diameter coverslips). Cells were cultured for one day on the gelatin matrix and then used 726 for experiments.

727 To analyse the degree of gelatin digestion of different cell lines, widefield microscopy 728 images were used. The labelled gelatin coat underneath each cell was manually evaluated 729 into no, low or high digestion dependent on the loss of fluorescent signal compared to the 730 undigested coat. For drug-induced disassembly of FAs on labelled gelatin matrix, the 
731 quantification of the switch from FAs to clathrin-coated plaques was performed as

732 described before. Additionally, each FA was evaluated manually for gelatin digestion.

\section{Production of 3D-micropatterns by soft lithography}

734 To prepare 3D-micropatterns suitable for microscopy, soft lithography with a PDMS master

735 and optically clear glue was performed. The protocol for PDMS master production was

736 adapted from Lücker et al., 201478 and the protocol for 3D-micropattern production with

737 optically clear glue from Ray et al., 201779.

738 To produce the PDMS master, diffraction gratings (Edmund Optics, \#54-510) with a groove 739 density of 500 grooves per $\mathrm{mm}$ were glued into a $10 \mathrm{~cm}$ petri dish using double-faced 740 adhesive tape. One clip-pack PDMS (SYLGARD 184, 10 g clip-pack; Sigma, \#761036) was 741 mixed, poured in the petri dish, and de-gased for 1 hour. The PDMS was cured at $50^{\circ} \mathrm{C}$ 742 overnight. The PDMS on the diffraction gratings was cut into small squares (approximately $7431 \times 1 \mathrm{~cm}$ ) and peeled off.

744 These PDMS masters were used to generate 3D-micropatterns made of Norland optical 745 adhesive (Norland Products, NOA73). A drop of NOA 73 was put on a coverslip and the 746 PDMS master was placed on top of this drop. NOA 73 was cured by UV light (UV Stratalinker 747 1800, Stratagene) for 15 minutes and the PDMS master was peeled from the coverslip 748 leaving behind the micropatterned optically clear NOA 73 film. The 3D-micropatterns were 749 illuminated with UV light a second time for 15 minutes to ensure completeness of curing. 750 These 3D-micropatterns were sterilized with ethanol before usage.

\section{Sequential seeding of cells on gridded coverslips}

752 80,000 cells were seeded in $\mu$-Dish $35 \mathrm{~mm}$ (high Grid-50 Glass Bottom; ibidi, 81148).

75312 hours after seeding, cells on grids were imaged by live-cell confocal microscopy.

754 Afterwards, cells were removed with 15 mM EDTA in PBS for 10 minutes. After the removal 755 of cells, the gridded coverslips were either left untreated, treated with $0.05 \%$ Trypsin/EDTA 756 for 20 minutes at $37^{\circ} \mathrm{C}$ or cleaned by sequential sonification in $1 \mathrm{M} \mathrm{KOH}$, acetone, ethanol, 757 and $\mathrm{H}_{2} \mathrm{O}$ for 10 minutes. Another round of cells was seeded as described above. Live-cell 758 confocal imaging was performed 12 hours after seeding at the positions imaged in the first

759 round of cells. To evaluate the amount of clathrin structures formed at the same position in 
760 both rounds of cells, the images were aligned with ImageJ/Fiji using the landmarks plugin.

761 Three landmarks from the grid were selected in both pictures and the second image was

762 registered to the first image with the Best Rigid Registration method. An automated image

763 analysis workflow by KNIME was used to perform object-based colocalization analysis of

764 clathrin structures for quantification. A binary mask for the AP2-eGFP signal in both images

765 was used to check for overlapping objects and to quantify the percentage from all clathrin

766 structures found in the first round of cells. Only clathrin structures at regions covered by

767 cells in both images were analysed.

\section{Tracking of clathrin structures}

769 For the tracking of CME events, we used ilastik (http://ilastik.org). First the images were

770 segmented using the pixel classification and object classification workflow. For tracking, the

771 automatic tracking workflow was used. The maximal distance was put to 5 to avoid merging

772 of close tracks. Tracking results were further analysed by automated workflows using

773 KNIME to calculate features (lifetime, maximal fluorescence intensity and average position)

774 of each CME event.

\section{Tracking of cell migration}

776 For the tracking of cell migration, we used the ImageJ/Fiji plugin Manual Tracking. The

777 position of a cell was manually defined by the centre of its cell body for each time point. The

778 tracking results were further analysed by automated workflows using KNIME to calculate

779 the directionality of cell migration. The directionality was defined as the angle between two

780 sequential positions of a cell. 


\section{Acknowledgments}

782 This work was supported by research grants from the Chica and Heinz Schaller Foundation

783 and the Deutsche Forschungsgemeinschaft (DFG) in TRR186 (project 09) to SB. DB was

784 supported by a fellowship from the Hartmut Hoffmann-Berling International Graduate

785 School of Molecular \& Cellular Biology (HBIGS) at the Heidelberg University, by a travel

786 collaboration grant from the Boehringer Ingelheim Fonds and from the DFG via the

787 SFB1129 (project 14). SB is members of the cluster of excellence CellNetworks. JWT is

788 supported by the Intramural Research Program of the US National Heart Lung and Blood

789 Institute (NHLBI), National Institutes of Health (NIH). We would like to thank Ulrike Engel

790 and the Nikon Imaging Center (Heidelberg University) for support with TIRF microscopy,

791 Vibor Laketa from the Department of Infectious Diseases, Virology (University Hospital

792 Heidelberg) for support with spinning disc microscopy, and the US National Heart Lung and

793 Blood Institute (NHLBI) Electron Microscopy Core and Light Microscopy Core facilities for

794 use of equipment.

\section{Author contributions}

796 DB designed and performed experiments, analysed data and wrote manuscript. MM 797 designed and established BacMam mammalian expression system. VS performed drug798 induced FA disassembly assays and designed 3D-micropattern experiments. CH designed, 799 performed and analysed AFM measurements. CZ and EACA designed adhesive micropattern 800 experiments. KAS and JWT designed and performed TEM and CLEM experiments. SB 801 supervised the project, designed experiments, interpreted data and wrote manuscript. The 802 authors declare that they do not have competing financial interests. 


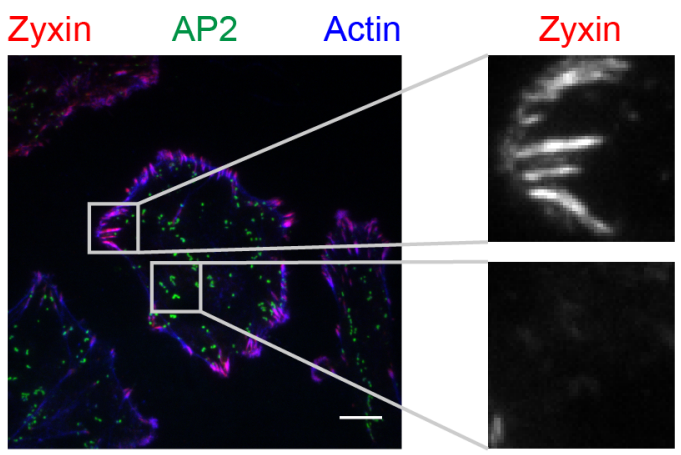

b
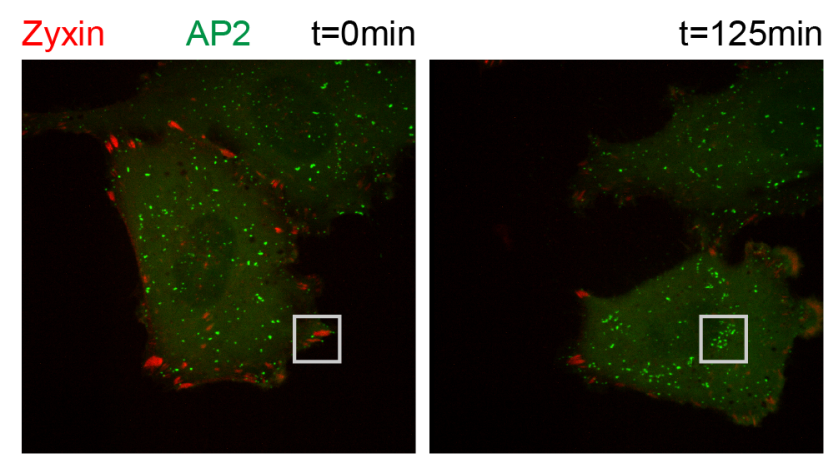

\section{Zyxin AP2}

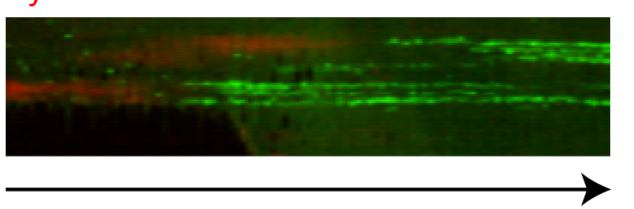

$\mathrm{t}=170 \mathrm{~min}$ d

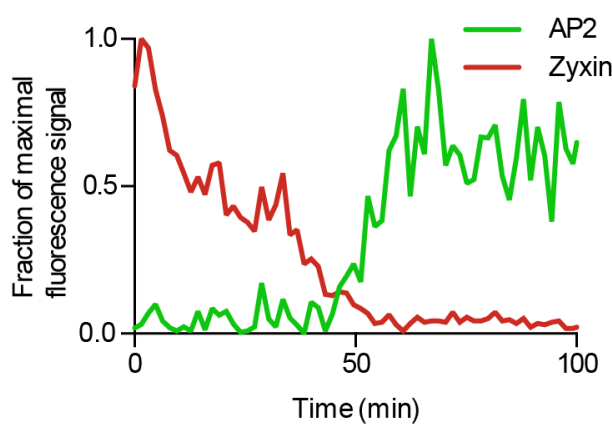

Actin
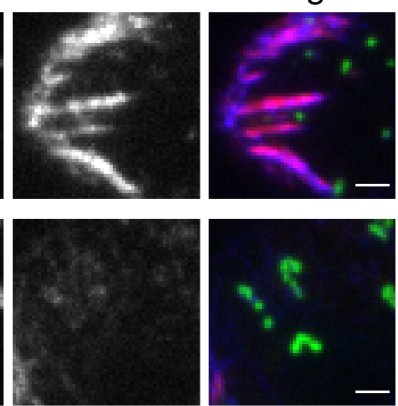

Zyxin mask $\mathrm{t}=0 \mathrm{~min}$ AP2 $\mathrm{t}=125 \mathrm{~min}$

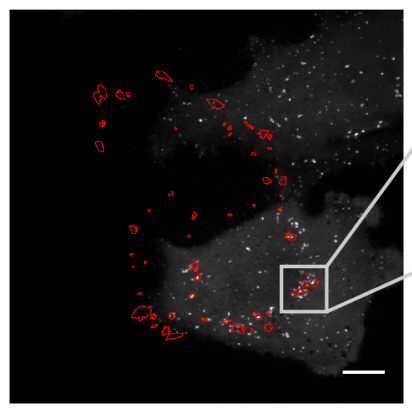

Zyxin AP2

Zyxin mask $\mathrm{t}=0$ min $\quad \mathrm{t}=125 \mathrm{~min}$ AP2 $\mathrm{t}=125 \mathrm{~min}$
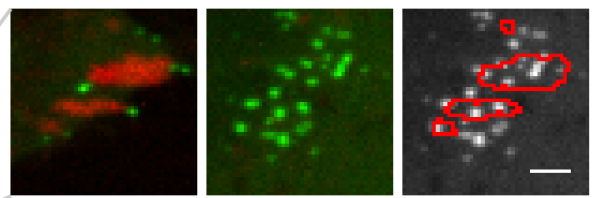

Switch from focal adhesions to clathrin-coated plaques

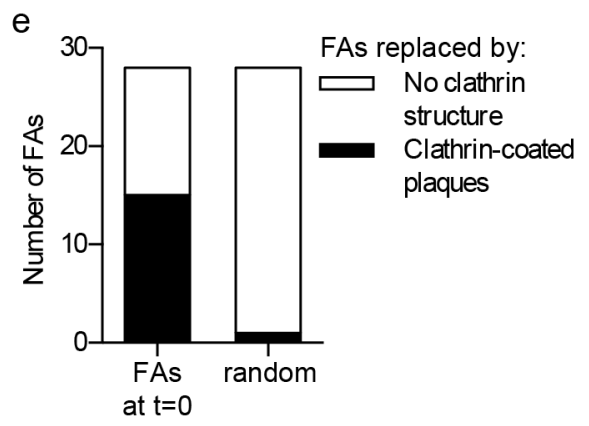

803 Figure 1: Switch from FAs to clathrin-coated plaques.

804 (a) Representative image from total internal reflection fluorescence (TIRF) microscopy of 805 U373 stably expressing AP2-eGFP (green) and transiently expressing mCherry-zyxin (red) 806 stained for actin with Alexa Fluor 647-labelled phalloidin (blue). Right: Zoom on FAs 807 labelled by mCherry-zyxin (upper panel) and on clathrin-coated plaques labelled by AP2-

808 eGFP (lower panel). (b) Live-cell confocal spinning disc microscopy of U373 stably 809 expressing AP2-eGFP (green) and transiently expressing mCherry-zyxin (red). Overview of 810 a representative migrating cell at time point 0 (left) and 125 minutes later (middle). 811 (Right) Merged images of a mask marking the mCherry-zyxin objects at time point 0 (red)

812 and the AP2-eGFP signal 125 minutes later (green). Right: Zoom on FAs that switch to 813 clathrin-coated plaques. (c) Kymograph of the switch from FAs to clathrin-coated plaques 
814 shown in b over 170 minutes. (d) Normalized fluorescence intensity profiles of AP2-eGFP

815 (green) and mCherry-zyxin (red) of the switch from FAs to clathrin-coated plaques shown

816 in b. (e) Quantification of the number of FAs being replaced by clathrin-coated plaques. Data

817 are from live experiment performed in b. FAs at time point 0 were followed over 2 hours to

818 quantify the number of FA replaced by clathrin-coated plaques (black) vs. the number of FA

819 not replaced by plaques (no clathrin structures (white)). As a control the appearance of

820 clathrin-coated plaques at randomly placed ROIs of the same size was analysed. Scale bars:

$82110 \mu \mathrm{m}$ (overviews) or $2 \mu \mathrm{m}$ (zooms). 
a $\alpha \vee \beta 5$ AP2 Merge
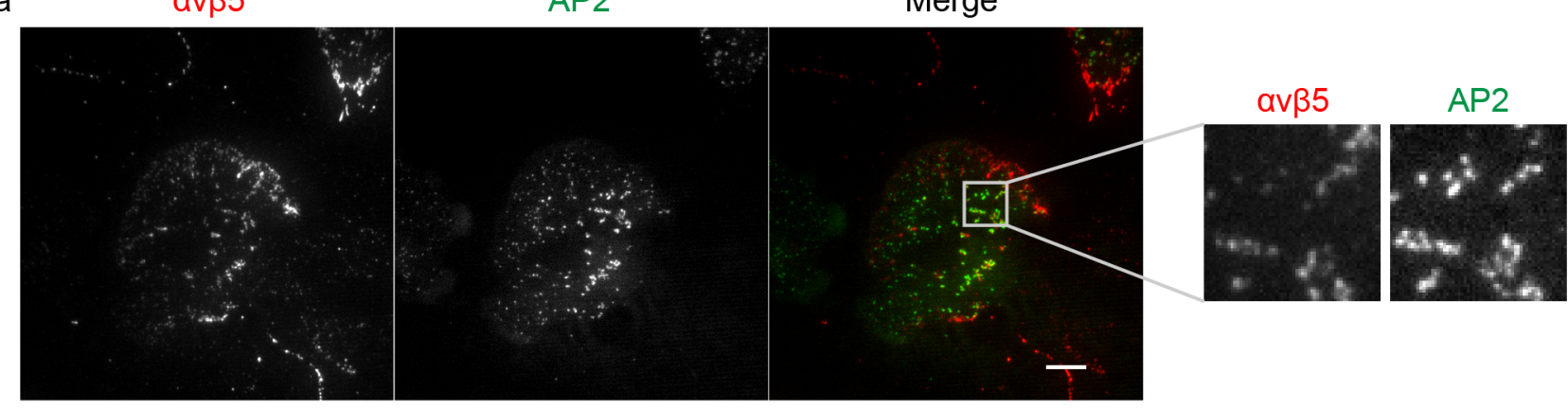

Merge

b

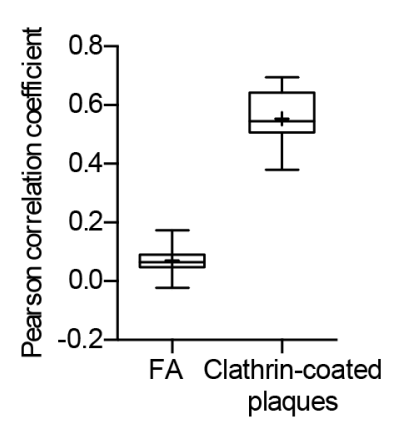

C $\mathrm{AP} 2$

before treatment

20min Cilengitide
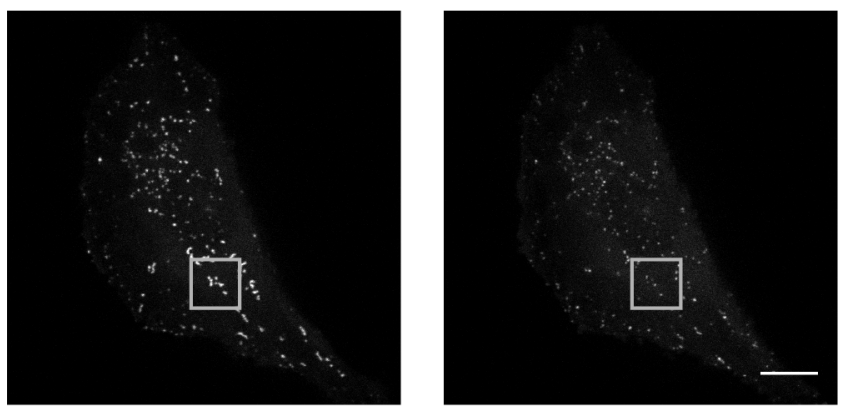

d

\section{Cilengitide} addition

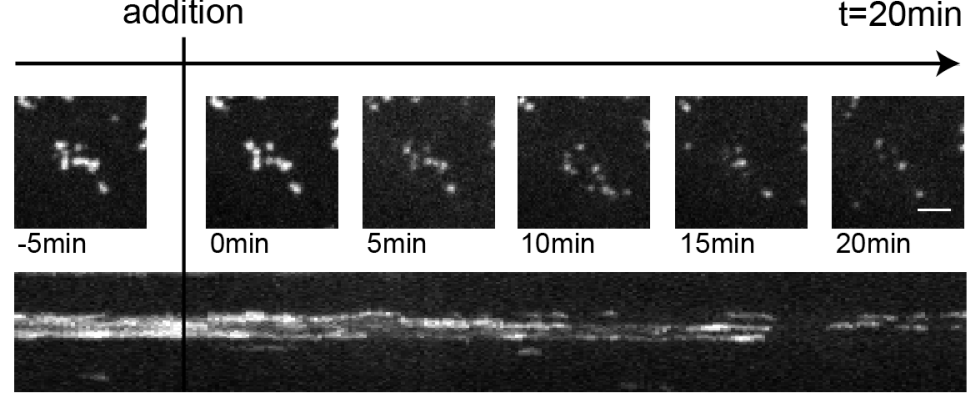

e

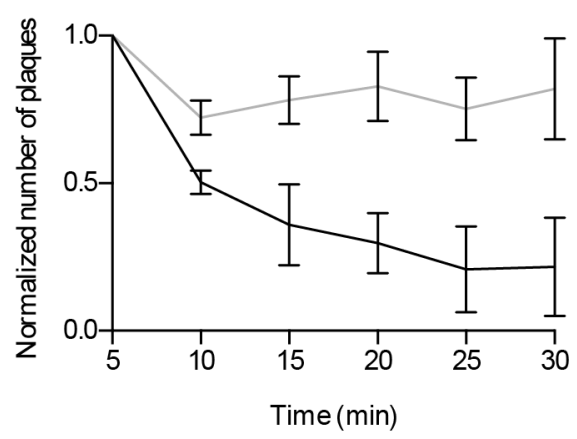

Cilengitide DMSO

823 (a) Representative image from TIRF microscopy of U373 stably expressing AP2-eGFP

824 (green) immunostained for integrin $\alpha v \beta 5$ (red). Right: Zoom on clathrin-coated plaques.

825 (b) Quantification of the colocalization between $\alpha \mathrm{v} \beta 5$ integrin and AP2-eGFP. The graph

826 shows the distribution of the Pearson correlation coefficient between AP2-eGFP and

827 integrin signals at either FAs or clathrin-coated plaques. Whiskers represent 10-90

828 percentile, box represents second and third quartile, line marks the median and cross the

829 mean. Results are computed from ten cells. (c) Live-cell spinning disc confocal microscopy

830 of U373 stably expressing AP2-eGFP treated with Cilengitide (10 $\mu \mathrm{M})$ for 20 minutes.

831 Shown is a representative cell before (left) and after 20 minutes of treatment with

832 Cilengitide (right). Clathrin-coated plaques were identified by monitoring the dynamic of

833 the clathrin structures before treatment with Cilengitide. (d) Serial view of a clathrin-coated 
834 plaque (ROI shown in b) (top panel) and corresponding kymograph (bottom). 835 (e) Quantification of the number of clathrin-coated plaques during Cilengitide (black) and 836 DMSO (grey) treatment. Number of plaques was normalized to the time at the beginning of 837 the treatment. Shown are the mean and SD calculated from four cells of each condition. 838 Scale bars: $10 \mu \mathrm{m}$ (overviews) or $2 \mu \mathrm{m}$ (zooms). 
a

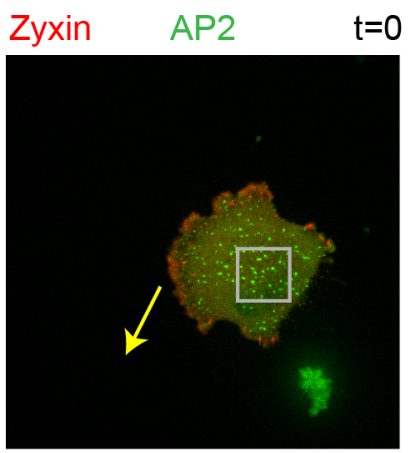

AP2 $\quad t=0 \quad t=4.4 \quad t=7.7 h$

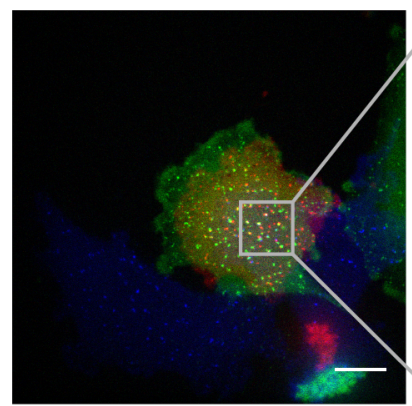

c First cell

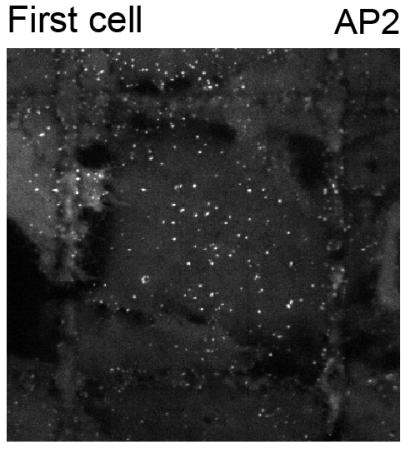

Grid

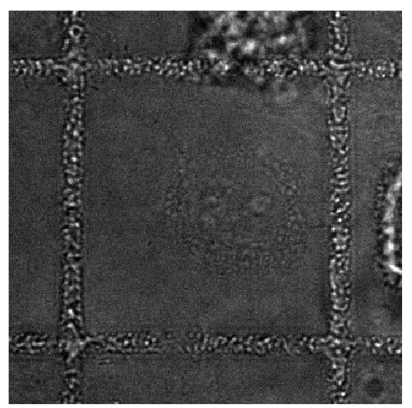

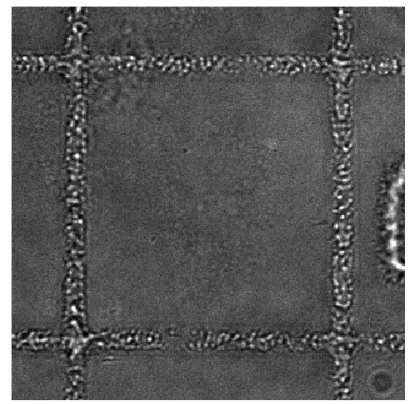

$t=3 h$

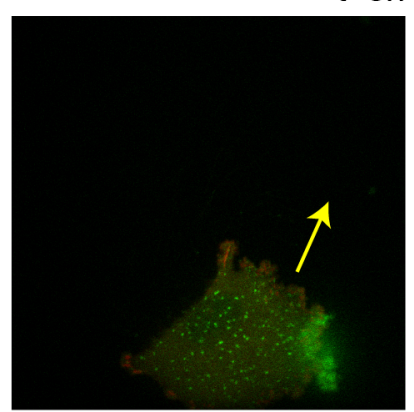

First cell

$(t=0)$

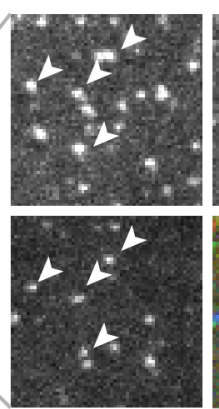

Second cell ( $\mathrm{t}=7.7 \mathrm{~h})$

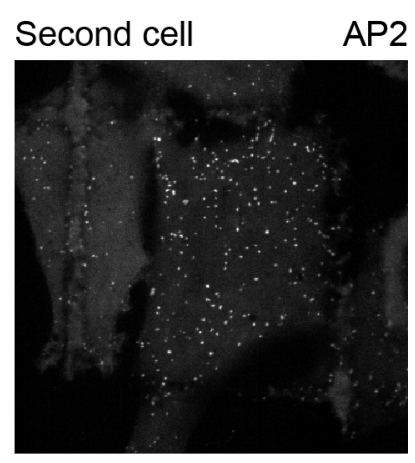

Grid

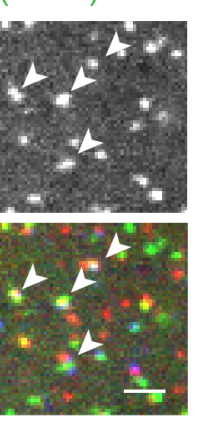

Merge

AP2
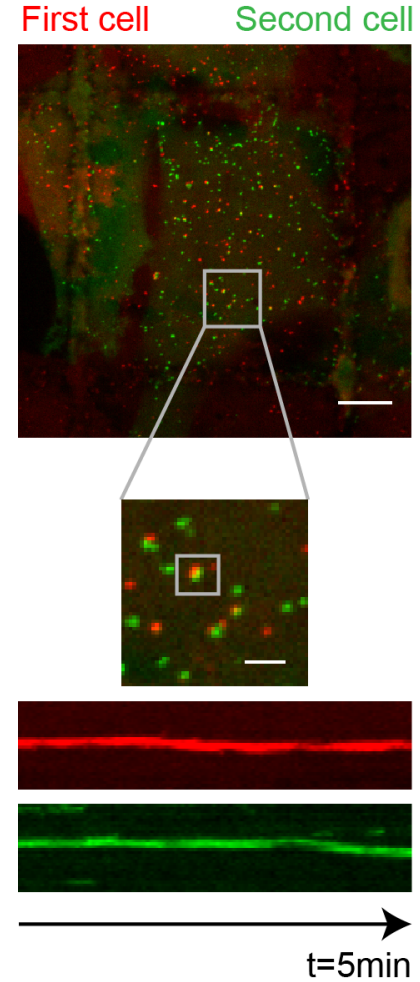

$t=4.4 h$

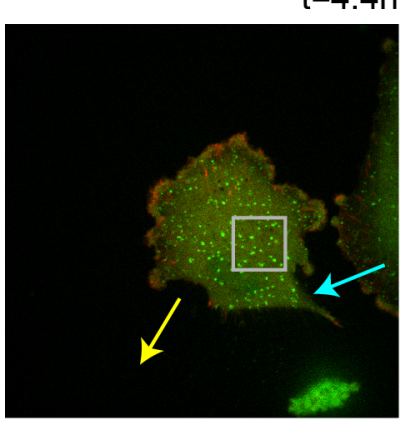

b Removing first cells
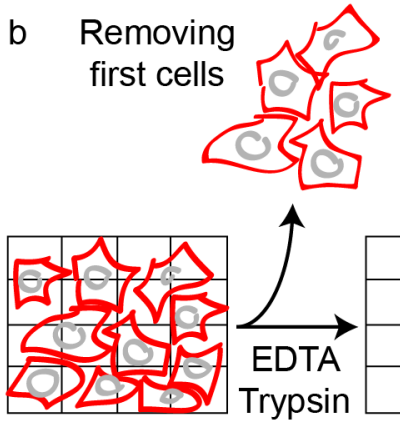

First cells

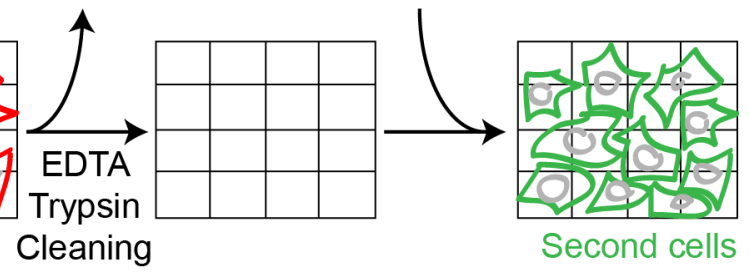

Cleaning

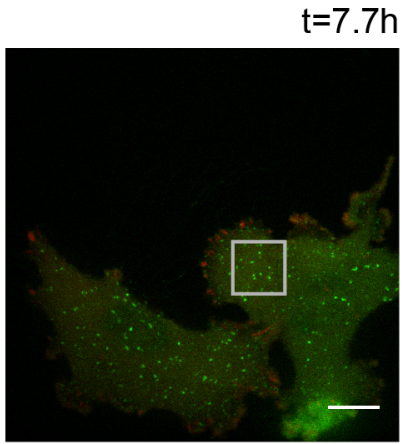

Seeding second cells

\section{AP2 overlap}
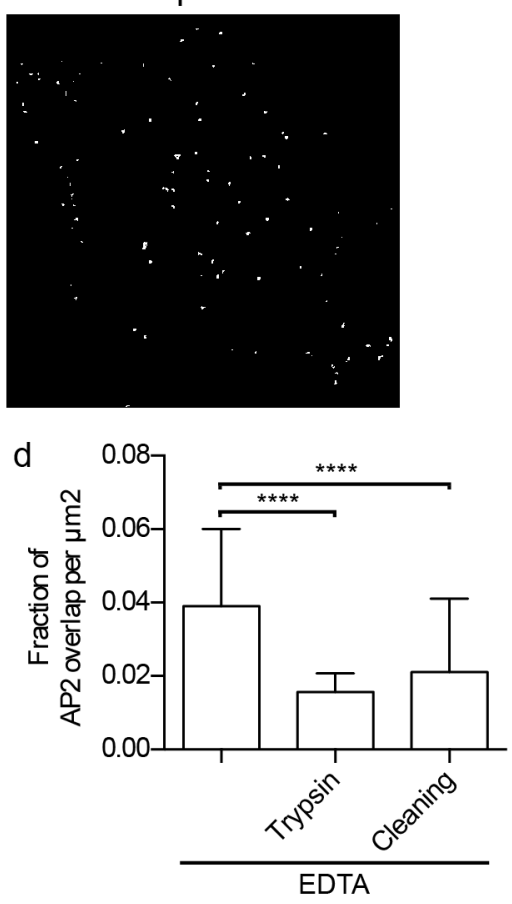

839 Figure 3: Extracellular signal induces clathrin-coated plaque formation. 
840 (a) Live-cell confocal spinning disc microscopy of U373 stably expressing AP2-eGFP (green)

841 and transiently expressing mCherry-zyxin (red). (Top) Snapshots of a time series of two

842 representative cells moving over the same area. Arrows point in the direction of cell

843 migration (first cell: yellow, second cell: blue). (Bottom left) Merged images of the AP2-

844 eGFP signal at three time points (first cell at $\mathrm{t}=0$ : red, first cell at $\mathrm{t}=4.4 \mathrm{~h}$ : green, second cell

845 at $\mathrm{t}=7.7 \mathrm{~h}$ : blue). Zoom on clathrin coats found at the same positions in all three time points

846 marked by arrow heads. (b) Schematic illustrating the sequence of the experiment using

847 gridded coverslips. A first round of cells (red) was imaged to identify the position of

848 clathrin-coated plaques and cells were then removed by EDTA solution. The gridded

849 coverslip was either left untreated or treated with trypsin or cleaned with a sequence of

$850 \mathrm{KOH}$, acetone, and ethanol to remove organic material. Afterwards a second round of

851 cells (green) was seeded on the same gridded coverslip and imaged to identify the position

852 of clathrin-coated plaques and to compare it with their position identified within the first

853 round of seeded cells. (c) Live-cell spinning disc confocal microscopy of two rounds of U373

854 stably expressing AP2-eGFP on the same position of a gridded coverslip with no treatment

855 before seeding the second round of cells. AP2-eGFP signal of first (top left) and second (top

856 middle left) round of cells. (Top middle right) Merged image of AP2-eGFP in first cell (red)

857 and second cell (green). Zoom in of representative overlapping clathrin coats from both

858 rounds of seeded cells and corresponding kymographs of 5 minute long movies of clathrin-

859 coated plaques from both rounds of seeded cells. (Top right) Binary mask of AP2-eGFP

860 overlap. (d) Quantification of overlapping clathrin coats in the first and second round of

861 cells using gridded coverslips. Shown are the mean with SD. Statistical analysis: t test,

862 number of analysed images: $\mathrm{n}=31$ (untreated), $\mathrm{n}=16$ (trypsin), and $\mathrm{n}=15$ (cleaning), $\mathrm{P}<0.01$.

863 Scale bars: $10 \mu \mathrm{m}$ (overviews), $2 \mu \mathrm{m}$ (zoom). 
bioRxiv preprint doi: https://doi.org/10.1101/493114; this version posted December 11,2018 . The copyright holder for this preprint (which was not certified by peer review) is the author/funder, who has granted bioRxiv a license to display the preprint in perpetuity. It is made available under aCC-BY-NC-ND 4.0 International license.

a

AP2 Gelatin
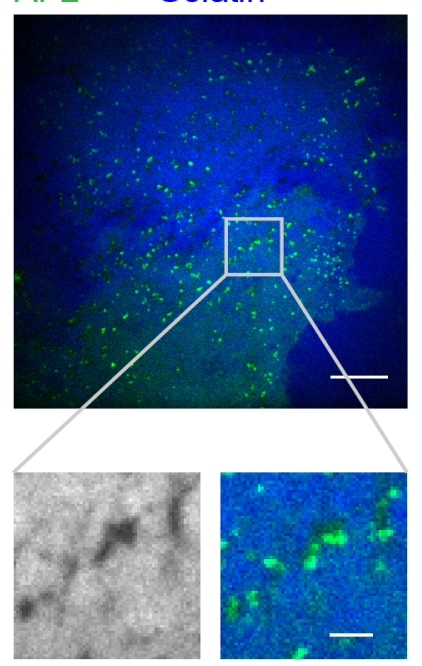

Gelatin

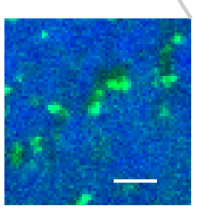

Merge b AP2

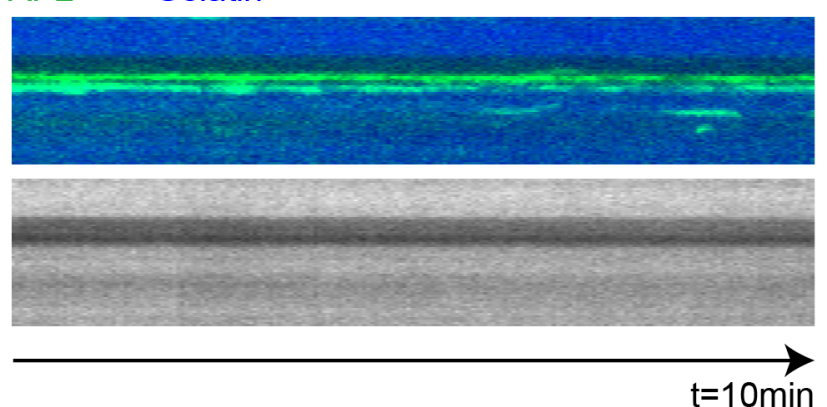

C

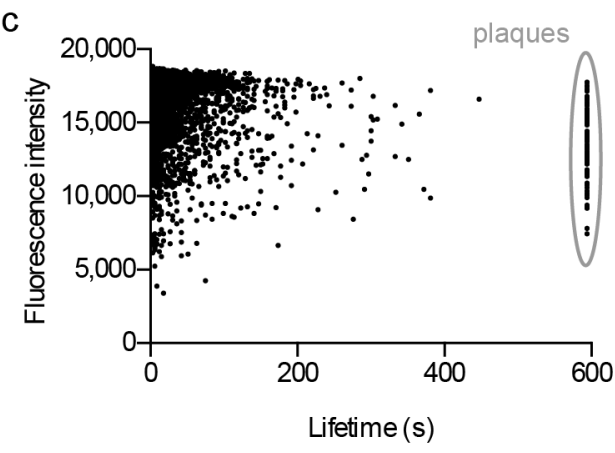

\section{Merge}

\section{Gelatin}

d

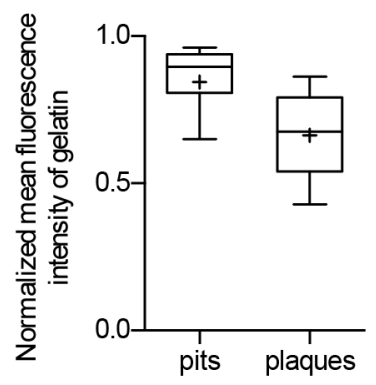

e

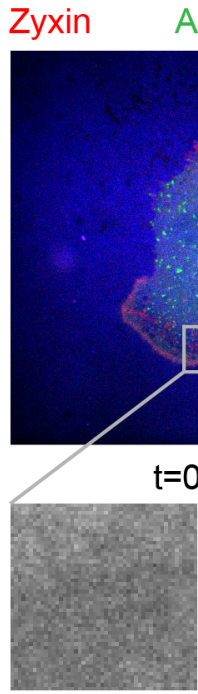

Gelatin
AP2 Gelatin

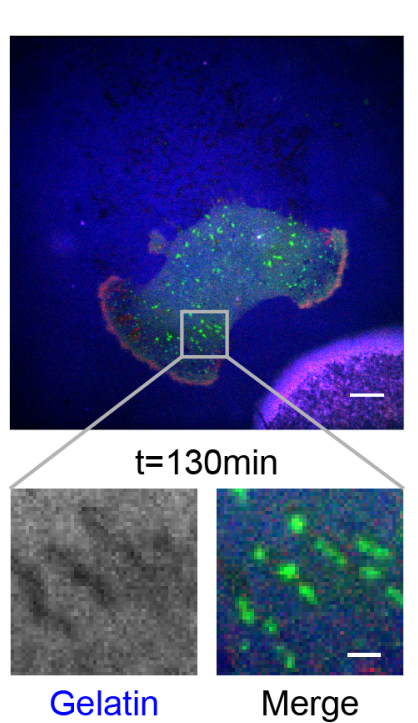

\section{f Zyxin}
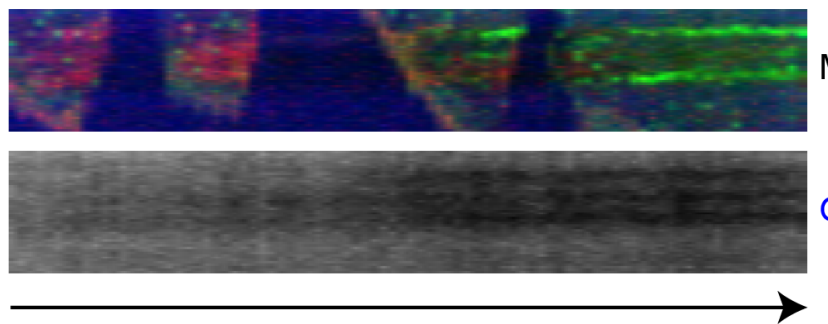

$t=130$ min

g

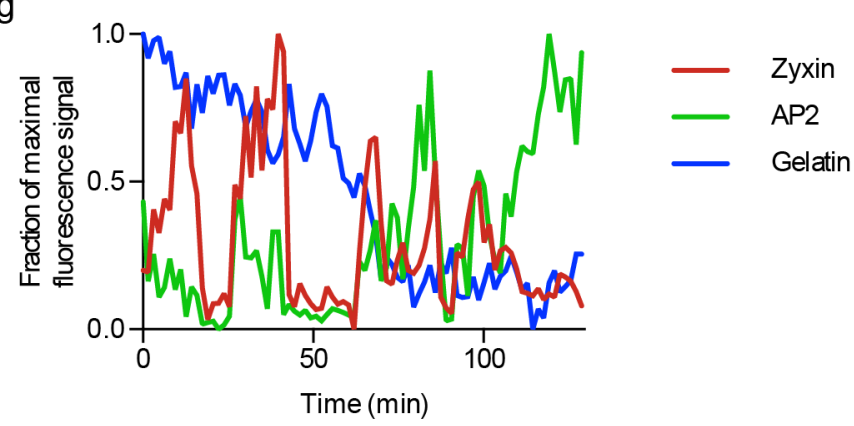

Gelatin

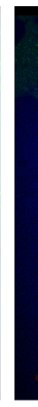

$t=120 \min$
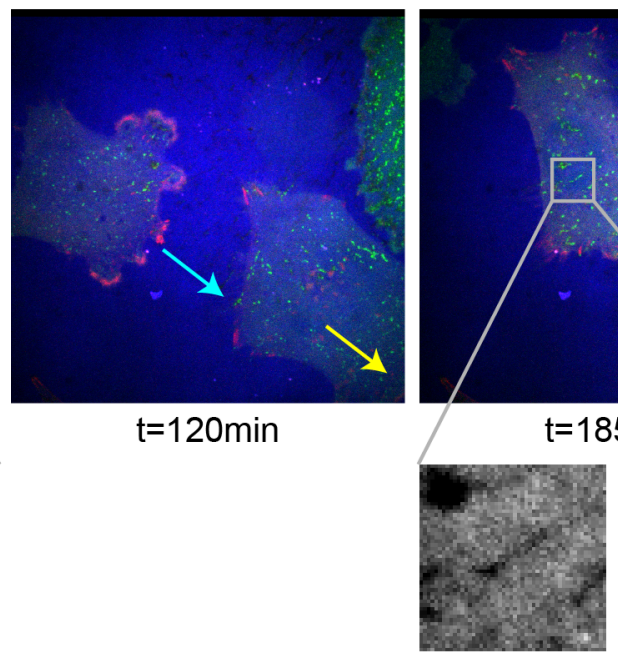

Gelatin

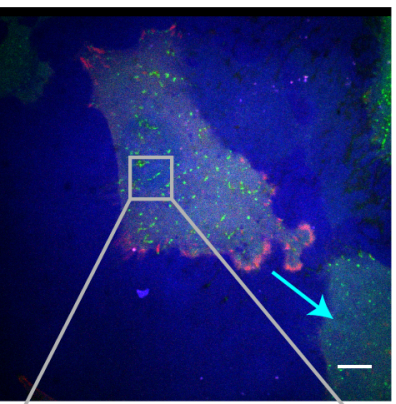

$\mathrm{t}=185 \mathrm{~min}$
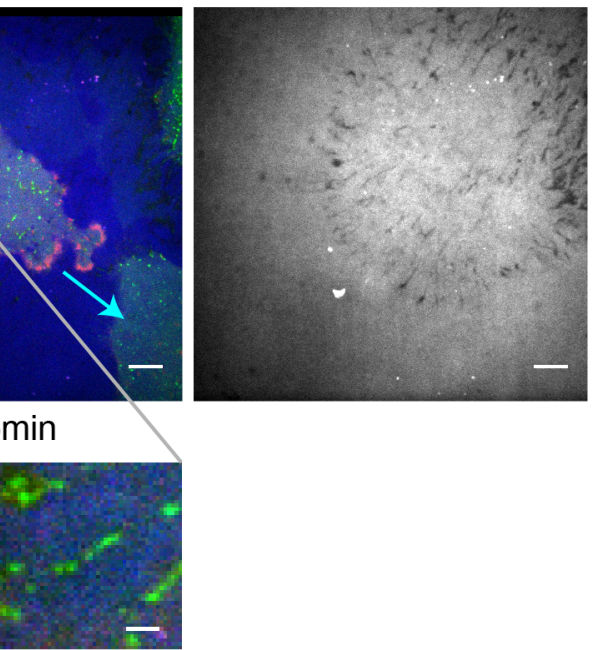

Merge
Merge

Gelatin h

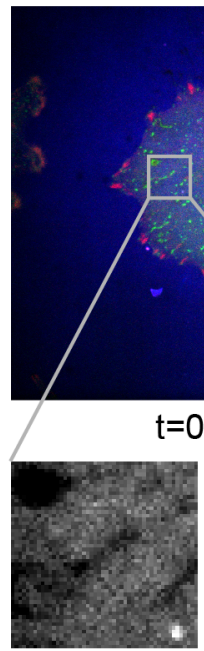

Gelatin 
865 Figure 4: Remodelled ECM as signal for clathrin-coated plaque formation.

866 (a) Live-cell confocal spinning disc microscopy of U373 stably expressing AP2-eGFP (green)

867 on coverslips coated with Alexa Fluor 647-labelled gelatin (blue). Right: Zoom in of clathrin-

868 coated plaques at digested gelatin spots. (b) Kymograph of clathrin-coated plaques and

869 gelatin digestion shown in an over 10 minutes. (c) Lifetime vs. mean fluorescence intensity

870 of clathrin tracks in U373 cells seeded on Alexa Fluor 647-labelled gelatin. Clathrin-coated

871 plaques are marked by a grey circle. Shown is a plot of a representative cell. Each dot

872 represents one CME track from a 10 minute long movie. (d) Distribution of mean

873 fluorescence intensity of Alexa Fluor 647-labelled gelatin at transient pits and at long-lived

874 clathrin-coated plaques ( $>10 \mathrm{~min})$ shown in c. Whiskers represent 10-90 percentile, box

875 represents second and third quartile, line marks the median and cross the mean. Results are

876 computed from 3,731 pits and 75 plaques. (e) Long-term live-cell confocal spinning disc

877 microscopy of U373 stably expressing AP2-eGFP (green) and transiently expressing

878 mCherry-zyxin (red) on coverslips coated with Alexa Fluor 647-labelled gelatin

879 (blue). Overview of a representative migrating cell at time point 0 (left) and 130 minutes

880 later (right). Bottom: Zoom in of FAs that switch to clathrin-coated plaques with the

881 corresponding gelatin signal (grey). (f) Kymograph of the switch from FAs to clathrin-

882 coated plaques on coverslips coated with Alexa Fluor 647-labelled gelatin shown in c over

883130 minutes. (g) Normalized fluorescence intensity profiles of AP2-eGFP (green), mCherry-

884 zyxin (red) and Alexa Fluor 647-labelled gelatin (blue) of the switch from FAs to clathrin-

885 coated plaques shown in c. (h) Two representative cells migrating over gelatin digestions.

886 Shown at time point 0 (left) and later time points (120 minutes (middle) and 185 minutes

887 (right)). Arrows point in the direction of migration (first cell: yellow; second cell: turquois).

888 Right: Overview of the Alexa Fluor 647-labelled gelatin signal (grey). Bottom: Zoom in time

889 points 0 and 185 minutes showing clathrin-coated plaques at the same gelatin digestions in

890 both cells. Scale bars: $10 \mu \mathrm{m}$ (overviews) or $2 \mu \mathrm{m}$ (zooms). 
bioRxiv preprint doi: https://doi.org/10.1101/493114; this version posted December 11,2018 . The copyright holder for this preprint (which was not certified by peer review) is the author/funder, who has granted bioRxiv a license to display the preprint in perpetuity. It is made available under ACC-BY-NC-ND 4.0 International license.

a Y-27632 treatment

Zyxin AP2 $\mathrm{t}=0 \mathrm{~min}$

Zyxin mask $\mathrm{t}=0 \mathrm{~min}$
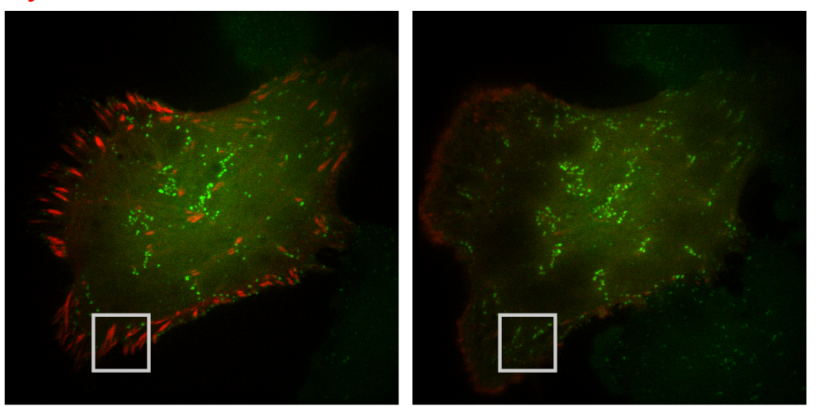

AP2 $t=30 \mathrm{~min}$

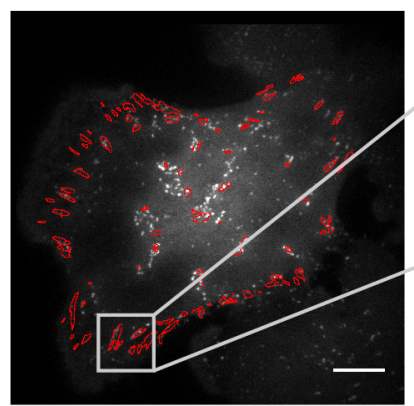

Zyxin AP2

Zyxin mask

$t=0$ min

b

\section{Zyxin AP2}

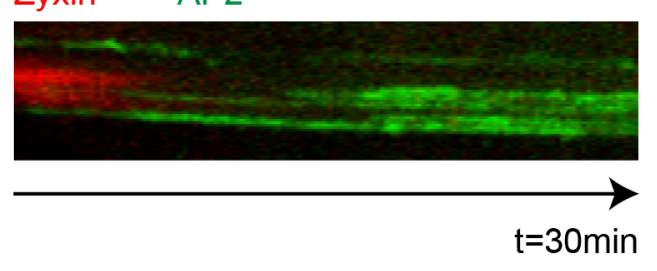

$\mathrm{t}=0 \mathrm{~min}$

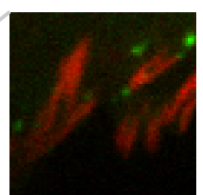

$t=30$ min
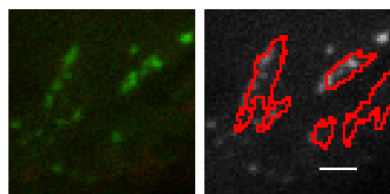

Drug-induced switch from focal adhesions to clathrin-coated plaques

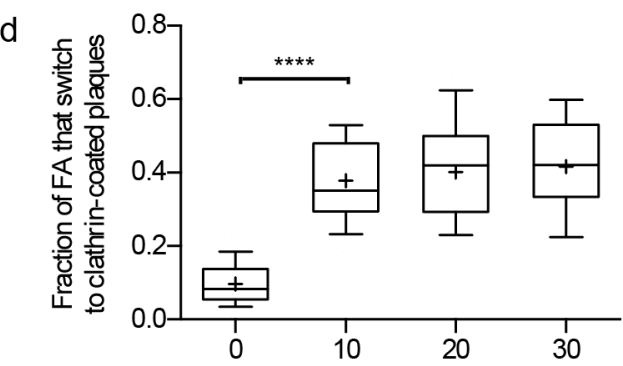

Time of Y-27632 treatment (min) e Y-27632 avß5

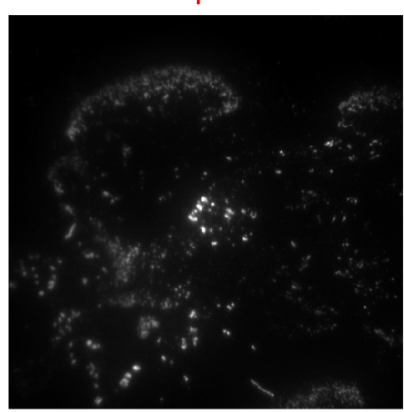

f

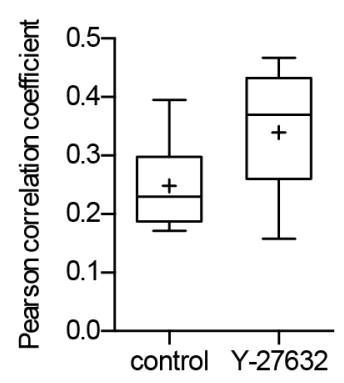

FAs replaced by:

$\simeq$ No clathrin structure

Long-lived plaques $(>10 \mathrm{~min})$

Transient clathrin structures (<10min)
AP2

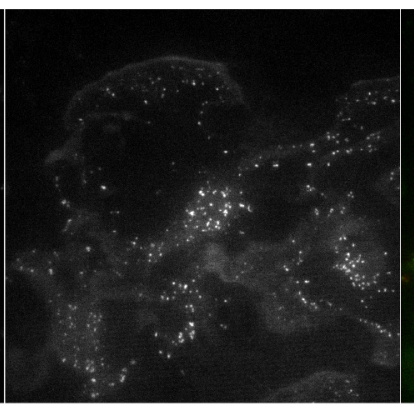

g Zyxin
Merge

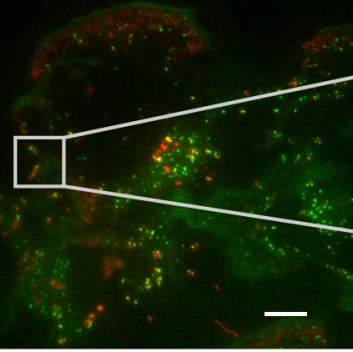

Y-27632

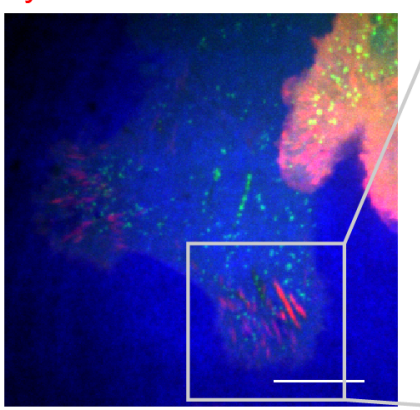

$\mathrm{t}=0 \mathrm{~min}$

$\mathrm{t}=30 \mathrm{~min}$

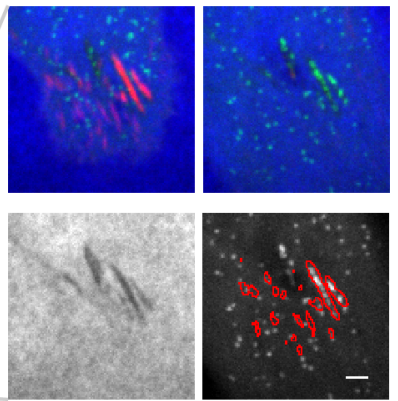

Gelatin Zyxin mask $\mathrm{t}=0 \mathrm{~min}$ AP2 $t=30$ min i WT

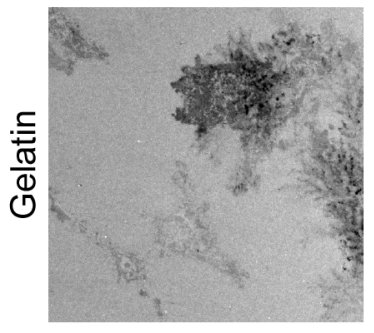

shMMP14

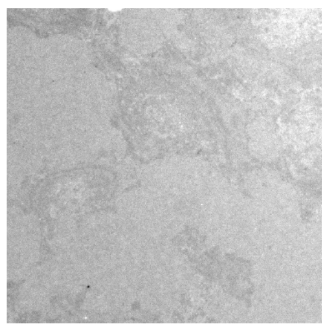

MMP14

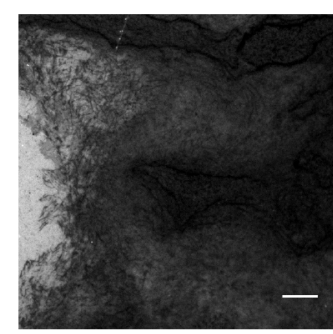

j $\square$ no $\square$ low $\square$ high k

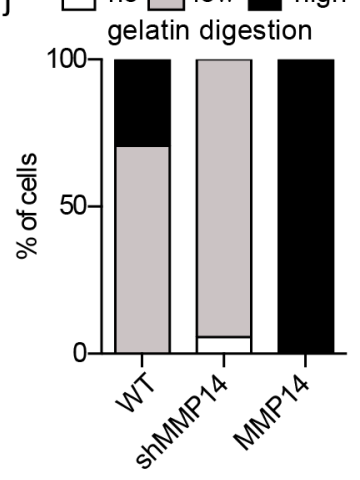

AP2
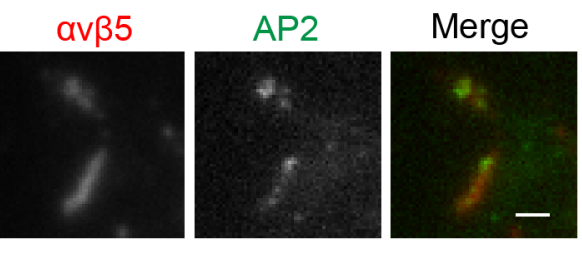

h

Y-27632

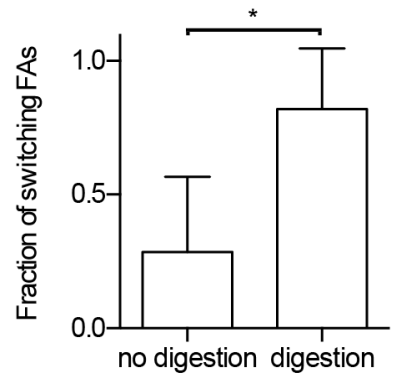

no digestion digestion 
893 (a) Live-cell confocal spinning disc microscopy of representative U373 cell stably 894 expressing AP2-eGFP (green) and transiently expressing mCherry-zyxin (red) treated with 895 Y-27632 $(10 \mu \mathrm{M})$. Cell before (left) and 30 minutes after drug treatment (middle). 896 (Right) Merged images of a mask marking the mCherry-zyxin objects before (red) and the 897 AP2-eGFP signal after Y-27632 treatment (green). Right: Zoom in of FAs that switch to 898 clathrin-coated plaques during the treatment. (b) Kymograph of the switch from FAs to 899 clathrin-coated plaques shown in b over 30 minutes of Y-27632 treatment. 900 (c) Quantification of FA replacement by clathrin structures during Y-27632 treatment 901 shown in a. Shown are the numbers of FAs that did not get replaced by clathrin structures 902 (white), that got replaced by transient (<10min) clathrin structures (grey) or long-lived 903 (>10min) clathrin-coated plaques (black). (d) Quantification of the switch from FAs to 904 clathrin-coated plaques during Y-27632 treatment. Whiskers represent 10-90 percentile, 905 box represents second and third quartile, line marks the median and cross the mean. 906 Results are computed from three repetitions. Statistical analysis: $t$ test, $\mathrm{n}=28, \mathrm{P}<0.01$. 907 (e) Representative image from TIRF microscopy of U373 stably expressing AP2-eGFP 908 (green) immunostained for integrin $\alpha v \beta 5$ (red) treated with Y-27632 for 20 minutes. Right: 909 Zoom in of clathrin-coated plaques at the periphery. Scale bars: $10 \mu \mathrm{m}$ (overviews) or $2 \mu \mathrm{m}$

910 (zoom). (f) Quantification of the colocalization between $\alpha v \beta 5$ integrin and AP2-eGFP. The 911 graph shows the distribution of the Pearson correlation coefficient between AP2-eGFP and 912 integrin signals in the whole cell either non treated (control) or treated with Y-27632 for 20

913 min. Whiskers represent 10-90 percentile, box represents second and third quartile, line 914 marks the median and cross the mean. Results are computed from ten cells. (g) Live-cell 915 confocal spinning disc microscopy of U373 cells stably expressing AP2-eGFP (green) and 916 transiently expressing mCherry-zyxin (red) on coverslips coated with Alexa Fluor 647917 labelled gelatin (grey) treated with Y-27632 $(10 \mu \mathrm{M})$. (Left) Overview of a representative 918 cell before treatment, (right) Zoom in of FAs that switch to clathrin-coated plaques during 919 the treatment on gelatin digestions. Scale bar: $10 \mu \mathrm{m}$ (overview), $2 \mu \mathrm{m}$ (zoom). 920 (h) Quantification of the percentage of switching FAs under Y-27632 treatment for FAs 921 without and with gelatin digestion. Shown is the mean with SD. Statistical analysis: paired $\mathrm{t}$ 922 test, $n=7, P<0.05$. (i) Images of widefield microscopy of coverslips coated with Alexa Fluor 923 647-labelled gelatin with representative digested areas generated by U373 wild-type (WT), 
924 stable knock down for MMP14 (shMMP14), or stable overexpression of MMP14 (MMP14)

925 on (red). Scale bar: $10 \mu \mathrm{m}$. (j) Analysis of the degree of gelatin digestion by U373 WT,

926 shMMP14 and MMP14. (k) Quantification of the switch from FAs to clathrin-coated plaques

927 after 20 minutes of Y-27632 treatment of U373 knock down for or overexpressing MMP14.

928 Whiskers represent 10-90 percentile, box represents second and third quartile, line marks

929 the median and cross the mean. Results are computed from three repetitions. Statistical

930 analysis: $\mathrm{t}$ test, $\mathrm{n}=28$ (shscr), $\mathrm{n}=50$ (shMMP14), and $\mathrm{n}=24$ (MMP14), $\mathrm{P}<0.05$. 
a

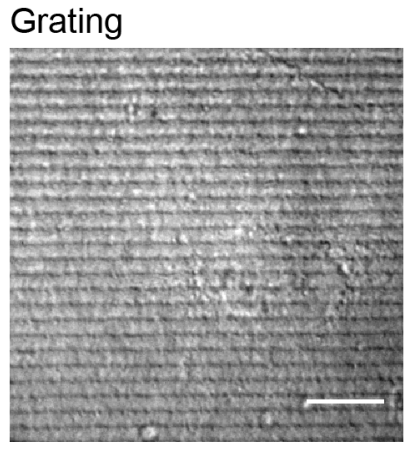

\section{AP2}

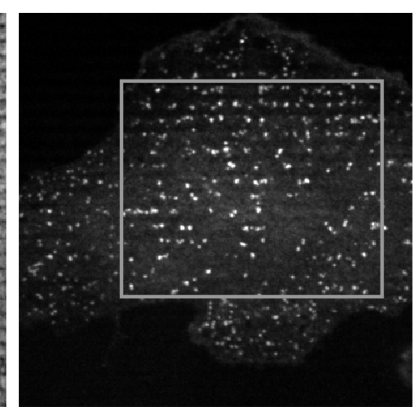

\section{Grating}

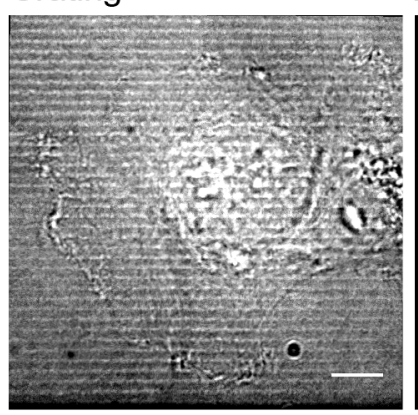

AP2

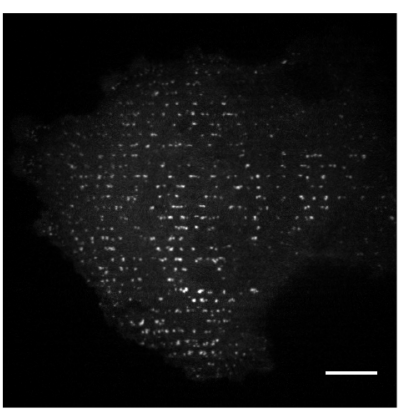

b AP2 c - AP2
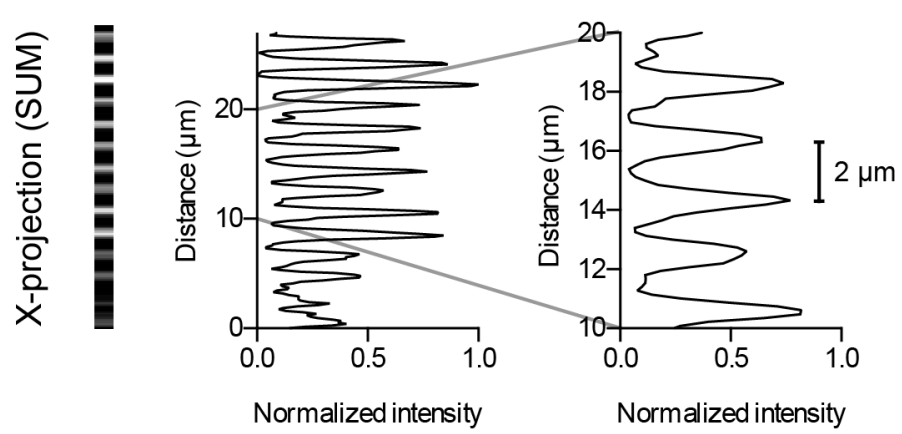

\section{Figure 6: Induction of clathrin-coated plaques by topographical cues.}

932 (a) Spinning disc confocal microscopy of U373 stably expressing AP2-eGFP (right) growing

933 on optically clear 3D-micropatterns (DIC, left). Box marks region used in b. (b) X-projection

934 of AP2-eGFP signal. (c) Left: Normalized intensity profile from the AP2-eGFP x-projection

935 shown in b. (d) Live-cell spinning disc confocal microscopy of U373 stably expressing AP2-

936 eGFP (middle) growing on optically clear 3D-micropatterns (DIC, left). (e) Left: Overview of

$937 \mathrm{x} / \mathrm{y}$-position of the tracking results from the cell shown in d. CME tracks were categories as

938 pits (transient, black dots) and plaques (persistent for > 10 minutes, red squares). CME

939 tracks were colour-coded according to their lifetime from yellow (0 s) to red (600 s). Scale

940 bars: $10 \mu \mathrm{m}$. 
a

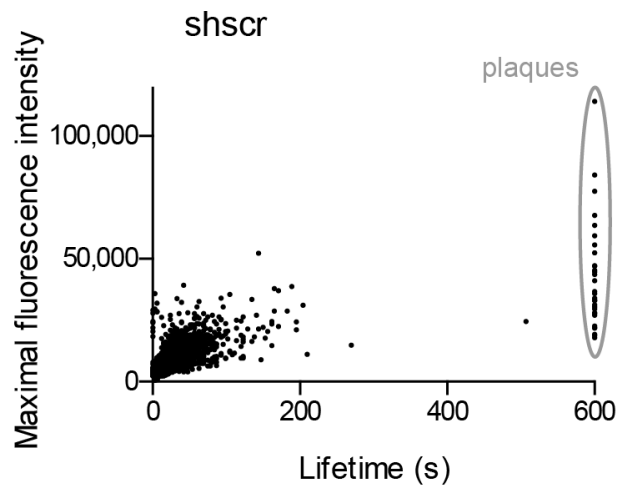

c Y-27632 treatment shEPS15/R

Zyxin AP2 $t=0$ min
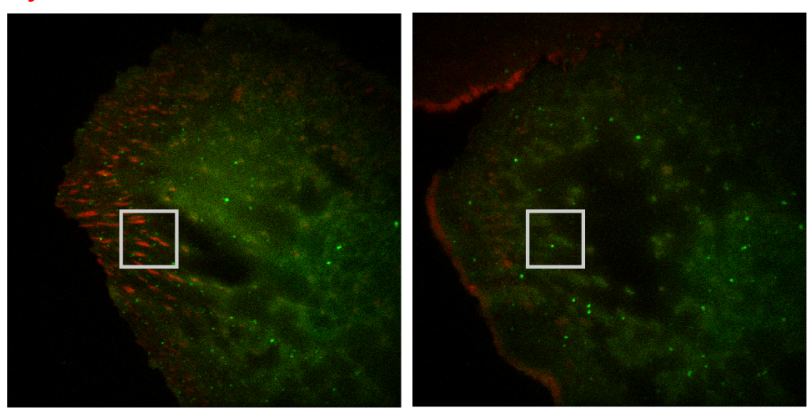

$t=30$ min
ShEPS15/R

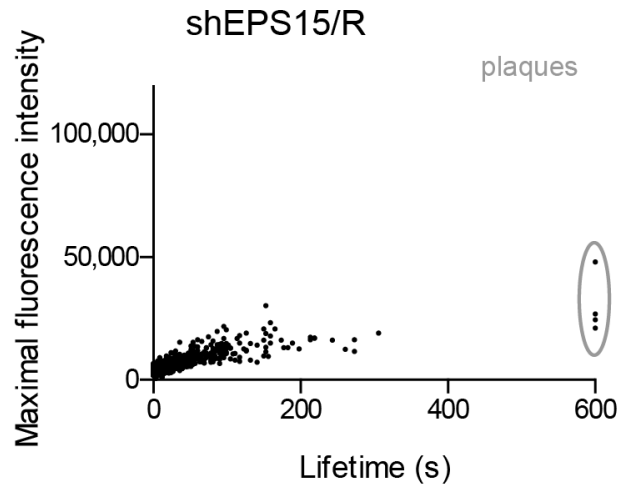

Zyxin mask $\mathrm{t}=0 \mathrm{~min}$ $\mathrm{AP} 2 \mathrm{t}=30 \mathrm{~min}$

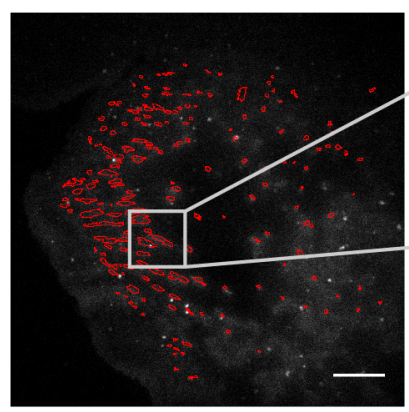

Zyxin AP2

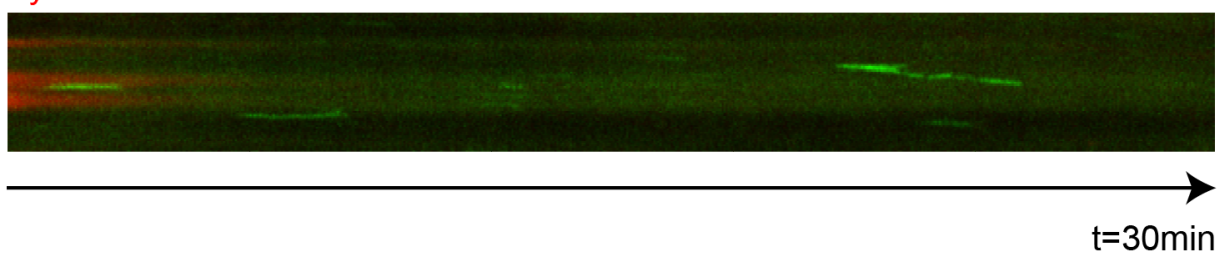

b

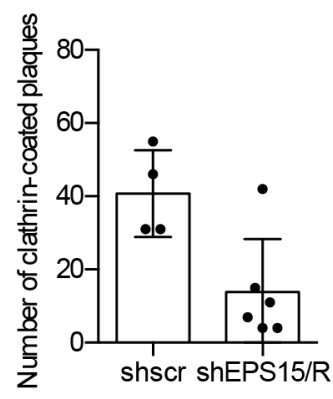

Zyxin mask

$\mathrm{t}=0 \mathrm{~min}$

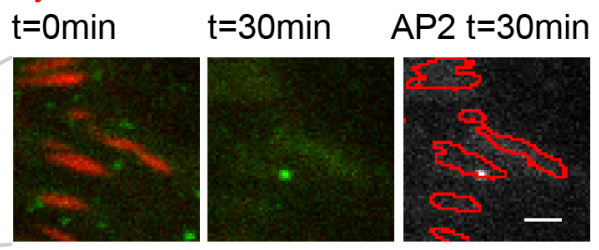

e

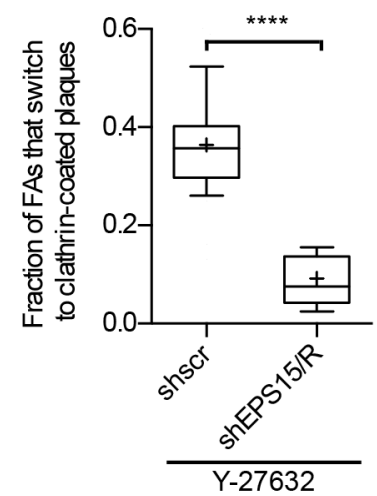

941 Figure 7: Eps15/R are regulators of clathrin-coated plaques formation.

942 (a) Lifetime versus maximal fluorescence intensity of AP2-eGFP of CME tracks for U373

943 stably expressing scrambled shRNA shscr (left) and shEPS15/R (right). Persistent clathrin-

944 coated plaques are marked by a grey circle. Shown are plots of a representative cell of each

945 cell line. Each dot represents one tracked CME event of a 10 minute long movie. (b)

946 Quantification of the number of clathrin-coated plaques in U373 stably expressing shscr and

947 shEPS15/R cells. Shown is the mean with SD computed from the tracking results of at least

948 four cells. Dots represent the results of each cell. (c) Live-cell confocal spinning disc

949 microscopy of representative U373 shESP15/R cell stably expressing AP2-eGFP (green) and

950 transiently expressing mCherry-zyxin (red) treated with Y-27632 (10 $\mu \mathrm{M})$. Cell before (left)

951 and 30 minutes after drug treatment (middle). (Right) Merged images of a mask marking 
952 the mCherry-zyxin objects before (red) and the AP2-eGFP signal after Y-27632 treatment

953 (green). Right: Zoom on FAs that disassemble during the treatment. (d) Kymograph of the

954 disassembly of FAs shown in b over 30 minutes of Y-27632 treatment. (e) Quantification of

955 the switch from FAs to clathrin-coated plaques after 20 minutes of Y-27632 treatment of

956 U373 shEPS15/R. Whiskers represent 10-90 percentile, box represents second and third

957 quartile, line marks the median and cross the mean. Results are computed from three

958 repetitions. Statistical analysis: $t$ test, $n=38$ (shscr), $n=29$ (shEPS15/R), $P<0.01$. Scale bars:

$95910 \mu \mathrm{m}$ (overviews) or $2 \mu \mathrm{m}$ (zooms). 


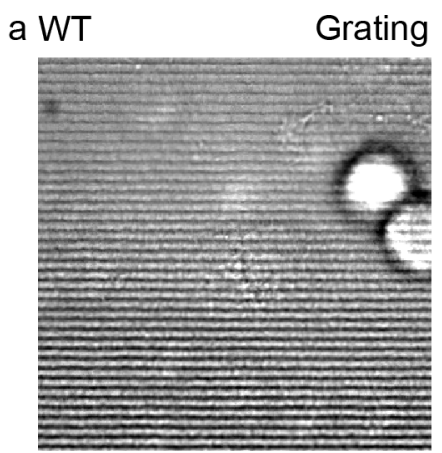

c WT

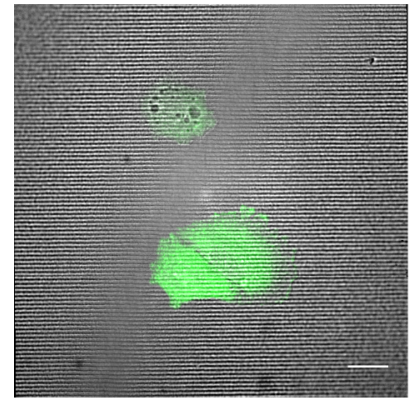

e

$$
\text { WT }
$$

0

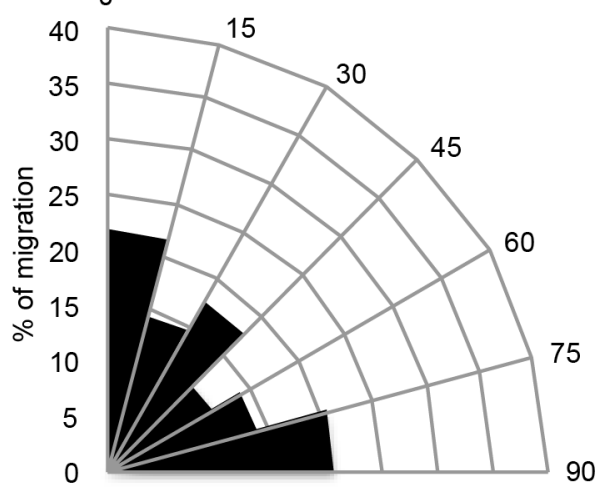

Directionality $\left({ }^{\circ}\right)$

f

shESP15/R flat

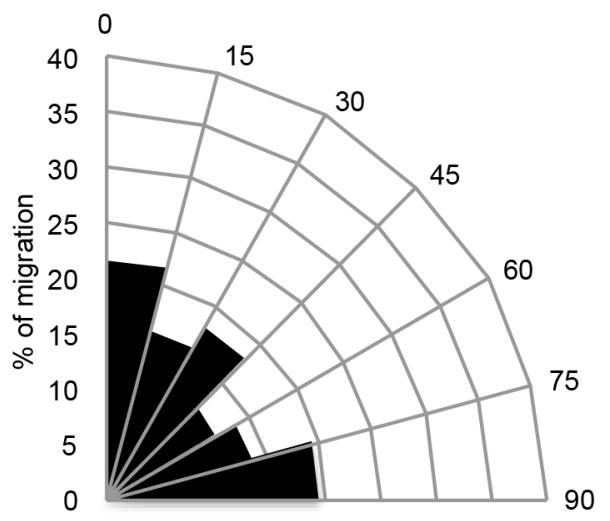

Directionality $\left(^{\circ}\right)$

flat
AP2

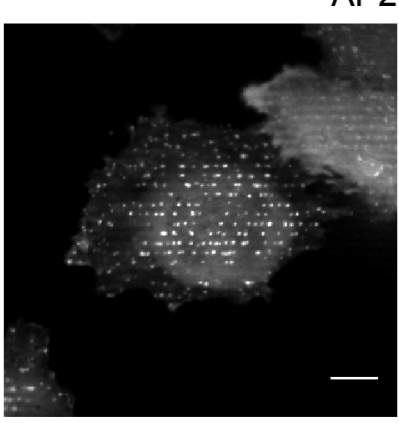

Tracks
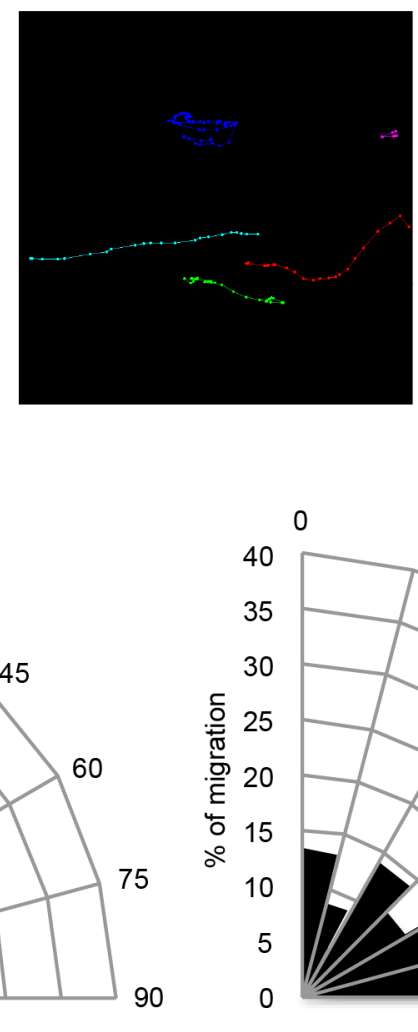

15

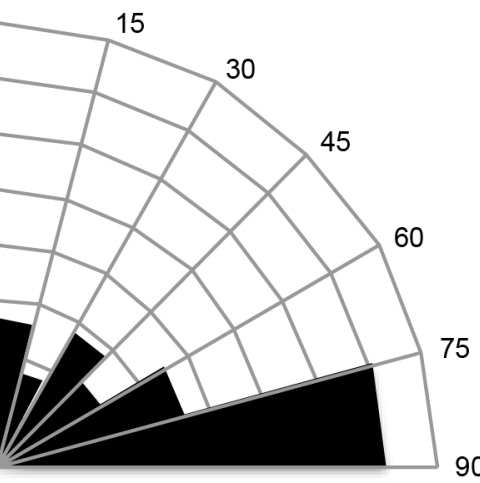

Directionality $\left({ }^{\circ}\right)$

grating

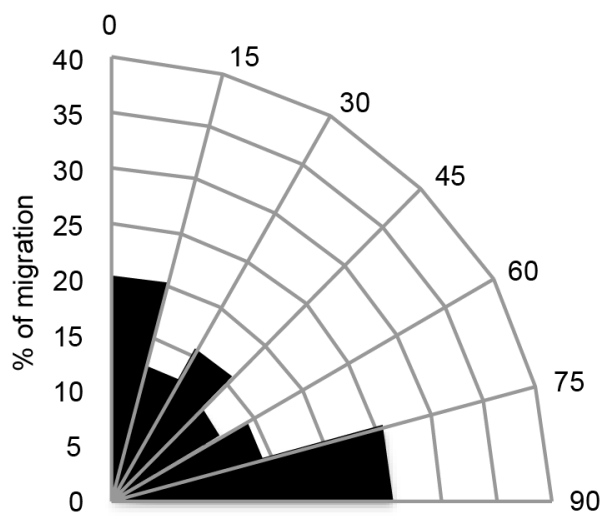

Directionality $\left({ }^{\circ}\right)$

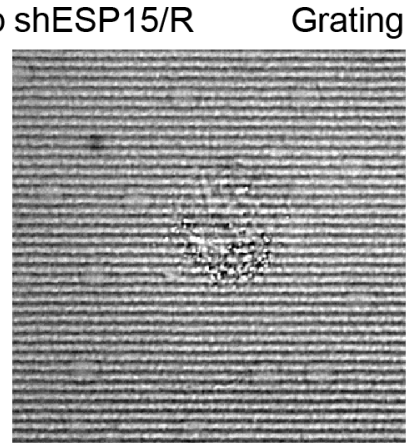

d shESP15/R AP2 Grating

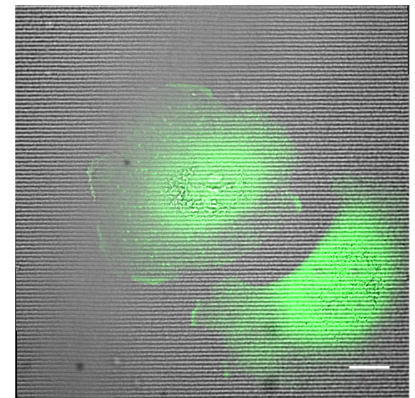

grating

g

AP2

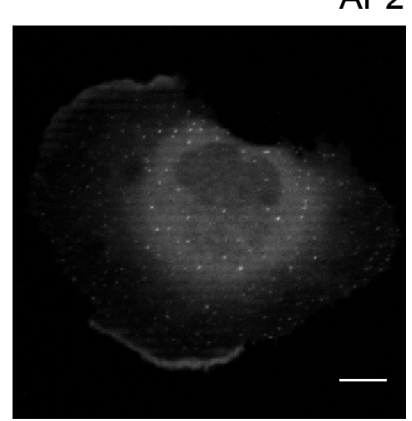

Tracks

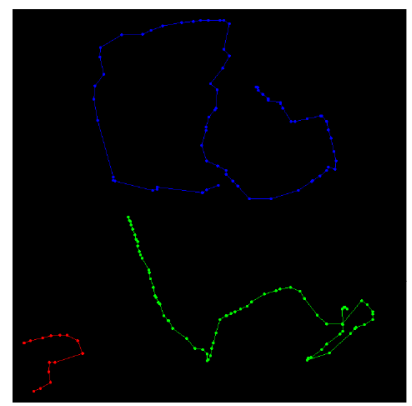

$\star \star \star \star *$

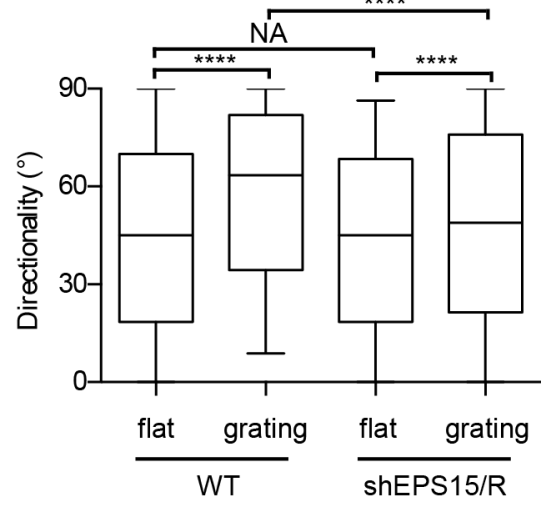

960 Figure 8: Clathrin-coated plaques establish directionality during contact guidance- 


\section{1 mediated cell migration.}

962 U373 WT (a) and shEPS15/R (b) stably expressing AP2-eGFP seeded on optically clear 3D-

963 micropattern. Scale bar: $10 \mu \mathrm{m}$. Live-cell spinning disc confocal microscopy of U373 WT (c)

964 and shEPS15/R (d) stably expressing AP2-eGFP (green) on optically clear 3D-micropattern

965 (grey, DIC). Migration tracks of cells are shown by multi-coloured dots and lines. Scale bar:

$96620 \mu \mathrm{m}$. Angular histogram of the directionality of migration for WT (e) and shEPS15/R (f)

967 cells on flat substrate (left) or grating (right). (g) Distribution of directionality of cells

968 migrating on flat substrate or 3D-micropattern. Whiskers represent 10-90 percentile, box

969 represents second and third quartile, and the line marks the median. Statistical analysis:

970 nonparametric $\mathrm{t}$ test (Mann-Whitney test), $\mathrm{P}<0.01$. Directionality was calculated between

971 time frames with a rate of 10 to 13 minutes. Results are computed from three repetitions.

972 Number of tracks: WT: $n=223$ (flat), $n=276$ (grating), shEPS15/R: $n=174$ (flat), $973 \mathrm{n}=155$ (grating). 


\section{References}

975 1. Frantz, C., Stewart, K. M. \& Weaver, V. M. The extracellular matrix at a glance. J. Cell

976 Sci. 123, 4195 LP-4200 (2010).

977 2. Hynes, R. O. \& Naba, A. Overview of the Matrisome-An Inventory of Extracellular

978 Matrix Constituents and Functions. Cold Spring Harb. Perspect. Biol. 4, a004903

979 (2012).

$980 \quad$ 3. Barczyk, M., Carracedo, S. \& Gullberg, D. Integrins. Cell Tissue Res. 339, 269-280

$981 \quad$ (2010).

982 4. Cavallaro, U. \& Dejana, E. Adhesion molecule signalling: not always a sticky business. 983 Nat. Rev. Mol. Cell Biol. 12, 189-97 (2011).

984 5. Horwitz, A. R. The origins of the molecular era of adhesion research. Nat. Rev. Mol.

$985 \quad$ Cell Biol. 13, 805-11 (2012).

986 6. Parsons, J. T., Horwitz, A. R. \& Schwartz, M. A. Cell adhesion: integrating cytoskeletal 987 dynamics and cellular tension. Nat. Rev. Mol. Cell Biol. 11, 633-643 (2010).

988 7. Geiger, B. \& Yamada, K. M. Molecular Architecture and Function of Matrix Adhesions. 989 Cold Spring Harb. Perspect. Biol. 3, a005033 (2011).

990 8. Sun, Z., Guo, S. S. \& Fässler, R. Integrin-mediated mechanotransduction. J. Cell Biol. $991 \quad 215,445-456(2016)$.

992 9. Gasiorowski, J. Z., Murphy, C. J. \& Nealey, P. F. Biophysical Cues and Cell Behavior: 993 The Big Impact of Little Things. Annu. Rev. Biomed. Eng. 15, 155-176 (2013).

994 10. Charras, G. \& Sahai, E. Physical influences of the extracellular environment on cell 995 migration. Nat. Rev. Mol. Cell Biol. 15, 813-24 (2014).

996 11. Engler, A. J., Sen, S., Sweeney, H. L. \& Discher, D. E. Matrix Elasticity Directs Stem Cell 
Lineage Specification. Cell 126, 677-689 (2006).

12. Ahmed, M. \& Ffrench-Constant, C. Extracellular Matrix Regulation of Stem Cell Behavior. Curr. Stem Cell Reports 2, 197-206 (2016).

13. Bonnans, C., Chou, J. \& Werb, Z. Remodelling the extracellular matrix in development and disease. Nat. Rev. Mol. Cell Biol. 15, 786-801 (2014).

14. Mouw, J. K., Ou, G. \& Weaver, V. M. Extracellular matrix assembly: a multiscale deconstruction. Nat. Rev. Mol. Cell Biol. 15, 771-785 (2014).

15. Singh, P., Carraher, C. \& Schwarzbauer, J. E. Assembly of Fibronectin Extracellular Matrix. Annu. Rev. Cell Dev. Biol. 26, 397-419 (2010).

16. Linder, S. \& Wiesner, C. Feel the force: Podosomes in mechanosensing. Exp. Cell Res. 343, 67-72 (2016).

17. Leckband, D. E. \& de Rooij, J. Cadherin Adhesion and Mechanotransduction. Annu. Rev. Cell Dev. Biol. 30, 291-315 (2014).

18. Galic, M. et al. External push and internal pull forces recruit curvature sensing N-BAR domain proteins to the plasma membrane. Nat. Cell Biol. 14, 874-881 (2012).

1012 19. Elkhatib, N. et al. Tubular clathrin/AP-2 lattices pinch collagen fibers to support 3D cell migration. Science (80-. ). 356, eaal4713 (2017).

1014 20. Zhao, W. et al. Nanoscale manipulation of membrane curvature for probing 1015 endocytosis in live cells. Nat. Nanotechnol. 12, 750-756 (2017).

1016 21. Fratini, M. et al. Surface Immobilization of Viruses and Nanoparticles Elucidates Early 1017 Events in Clathrin-Mediated Endocytosis. ACS Infect. Dis. (2018).

1018 doi:10.1021/acsinfecdis.8b00134

1019 22. McMahon, H. T. \& Boucrot, E. Molecular mechanism and physiological functions of 
clathrin-mediated endocytosis. Nat. Rev. Mol. Cell Biol. 12, 517-533 (2011).

1021 23. Kaksonen, M. \& Roux, A. Mechanisms of clathrin-mediated endocytosis. Nat. Rev. Mol. $1022 \quad$ Cell Biol. 19, 313 (2018).

1023 24. Avinoam, O., Schorb, M., Beese, C. J., Briggs, J. A. G. \& Kaksonen, M. Endocytic sites mature by continuous bending and remodeling of the clathrin coat. Science (80-. ). 348, 1369 LP-1372 (2015).

25. Scott, B. L. et al. Membrane bending occurs at all stages of clathrin-coat assembly and defines endocytic dynamics. Nat. Commun. 9, 419 (2018).

26. Bucher, D. et al. Clathrin-adaptor ratio and membrane tension regulate the flat-tocurved transition of the clathrin coat during endocytosis. Nat. Commun. 9, 1109 (2018).

27. Lampe, M., Vassilopoulos, S. \& Merrifield, C. Clathrin coated pits, plaques and adhesion. J. Struct. Biol. 196, 48-56 (2016).

1033 28. Merrifield, C. J., Perrais, D. \& Zenisek, D. Coupling between clathrin-coated-pit invagination, cortactin recruitment, and membrane scission observed in live cells. Cell 121, 593-606 (2005).

29. Grove, J. et al. Flat clathrin lattices: stable features of the plasma membrane. Mol. Biol. Cell 25, 3581-3594 (2014). clathrin-mediated endocytosis and signalling of specific receptors. Nat. Commun. 8, 16068 (2017).

1041 31. Maupin, P. \& Pollard, T. D. Improved preservation and staining of HeLa cell actin filaments, clathrin-coated membranes, and other cytoplasmic structures by tannic acid-glutaraldehyde-saponin fixation. J. Cell Biol. 96, 51-62 (1983). 
1044 32. De Deyne, P. G. et al. The vitronectin receptor associates with clathrin-coated

1045 membrane domains via the cytoplasmic domain of its beta5 subunit. J. Cell Sci. 111, 2729 LP-2740 (1998).

1047 33. Baschieri, F. et al. Frustrated endocytosis controls contractility-independent mechanotransduction at clathrin-coated structures. Nat. Commun. 9, 3825 (2018).

1049 34. Zuidema, A. et al. Mechanisms of integrin $\alpha \mathrm{V} \beta 5$ clustering in flat clathrin lattices. J. Cell Sci. (2018).

35. Heuser, J. Three-dimensional visualization of coated vesicle formation in fibroblasts. J. Cell Biol. 84, 560-583 (1980).

36. Saffarian, S., Cocucci, E. \& Kirchhausen, T. Distinct Dynamics of Endocytic ClathrinCoated Pits and Coated Plaques. PLoS Biol. 7, e1000191 (2009).

37. Kural, C. et al. Asymmetric formation of coated pits on dorsal and ventral surfaces at the leading edges of motile cells and on protrusions of immobile cells. Mol. Biol. Cell 26, 2044-2053 (2015).

38. Caswell, P. T., Vadrevu, S. \& Norman, J. C. Integrins: masters and slaves of endocytic transport. Nat. Rev. Mol. Cell Biol. 10, 843 (2009).

1060 39. Ezratty, E. J., Bertaux, C., Marcantonio, E. E. \& Gundersen, G. G. Clathrin mediates integrin endocytosis for focal adhesion disassembly in migrating cells. J. Cell Biol. 187, 733-747 (2009).

40. Chao, W.-T. \& Kunz, J. Focal adhesion disassembly requires clathrin-dependent endocytosis of integrins. FEBS Lett. 583, 1337-1343 (2009).

1065 41. Dechantsreiter, M. A. et al. N-Methylated Cyclic RGD Peptides as Highly Active and 1066 Selective $\alpha$ Vß3 Integrin Antagonists. J. Med. Chem. 42, 3033-3040 (1999).

1067 42. Mas-Moruno, C., Rechenmacher, F. \& Kessler, H. Cilengitide: The First Anti- 
Angiogenic Small Molecule Drug Candidate. Design, Synthesis and Clinical Evaluation. Anticancer. Agents Med. Chem. 10, 753-768 (2010).

43. Wang, Y. \& McNiven, M. A. Invasive matrix degradation at focal adhesions occurs via protease recruitment by a FAK-p130Cas complex. J. Cell Biol. 196, 375-385 (2012).

44. Riento, K. \& Ridley, A. J. ROCKs: multifunctional kinases in cell behaviour. Nat. Rev. Mol. Cell Biol. 4, 446-56 (2003).

45. Vicente-Manzanares, M., Choi, C. K. \& Horwitz, A. R. Integrins in cell migration - the actin connection. J. Cell Sci. 122, 199 LP-206 (2009).

46. Sato, H., Takino, T. \& Miyamori, H. Roles of membrane-type matrix metalloproteinase-1 in tumor invasion and metastasis. Cancer Sci. 96, 212-217

47. Itoh, Y. MT1-MMP: A key regulator of cell migration in tissue. IUBMB Life 58, 589(2005).

48. Takino, T. et al. Membrane-type 1 matrix metalloproteinase modulates focal adhesion stability and cell migration. Exp. Cell Res. 312, 1381-1389 (2006). promotes muscle cell migration and differentiation. Am. J. Pathol. 174, 541-549 (2009). Biol. 23, 16-21 (2013). extracellular matrix homeostasis. Nat. Rev. Mol. Cell Biol. 15, 802-812 (2014). mechanotransduction in cell biology. Nat. Rev. Mol. Cell Biol. 15, 825-33 (2014). 
$1092 \quad 53 . \quad$ Clark, P., Connolly, P., Curtis, A. S., Dow, J. A. \& Wilkinson, C. D. Topographical control of cell behaviour: II. Multiple grooved substrata. Development 108, 635 LP-644 (1990).

1095 54. Clark, P., Connolly, P., Curtis, A. S., Dow, J. A. \& Wilkinson, C. D. Cell guidance by ultrafine topography in vitro. J. Cell Sci. 99, 73 LP-77 (1991).

55. Petrie, R. J., Doyle, A. D. \& Yamada, K. M. Random versus directionally persistent cell migration. Nat. Rev. Mol. Cell Biol. 10, 538-549 (2009).

56. Kim, D.-H. et al. Mechanosensitivity of fibroblast cell shape and movement to anisotropic substratum topography gradients. Biomaterials 30, 5433-5444 (2009).

1101 57. Tamiello, C., Buskermolen, A. B. C., Baaijens, F. P. T., Broers, J. L. V \& Bouten, C. V. C. Heading in the Right Direction: Understanding Cellular Orientation Responses to Complex Biophysical Environments. Cell. Mol. Bioeng. 9, 12-37 (2016).

1104 58. Mulkearns, E. E. \& Cooper, J. A. FCH domain only-2 organizes clathrin-coated 1105 structures and interacts with Disabled-2 for low-density lipoprotein receptor 1106 endocytosis. Mol. Biol. Cell 23, 1330-1342 (2012).

59. Umasankar, P. K. et al. A clathrin coat assembly role for the muniscin protein central linker revealed by TALEN-mediated gene editing. Elife 3, (2014). Adaptor for Cargo Binding. Dev. Cell 37, 428-443 (2016). activators of the AP2 clathrin adaptor complex. Elife 3, e03648 (2014).

1113 62. Wang, L., Johnson, A., Hanna, M. \& Audhya, A. Eps15 membrane-binding and -bending activity acts redundantly with Fcho1 during clathrin-mediated endocytosis. Mol. Biol. Cell 27, 2675-2687 (2016). 
1116 63. Kim, D.-H., Provenzano, P. P., Smith, C. L. \& Levchenko, A. Matrix nanotopography as a regulator of cell function. J. Cell Biol. 197, 351-360 (2012).

1118 64. Nakatsuji, N. \& Johnson, K. E. Cell locomotion in vitro by Xenopus laevis gastrula mesodermal cells. Cell Motil. 2, 149-161 (1982).

65. Nakatsuji, N. \& Johnson, K. E. Ectodermal fragments from normal frog gastrulae condition substrata to support normal and hybrid mesodermal cell migration in vitro. J. Cell Sci. 68, 49 LP-67 (1984).

66. Nakatsuji, N. \& E. Johnson, K. Experimental manipulation of a contact guidance system in amphibian gastrulation by mechanical tension. Nature 307, 453-455 (1984).

67. Provenzano, P. P. et al. Collagen reorganization at the tumor-stromal interface facilitates local invasion. BMC Med. 4, 38 (2006).

68. Carey, S. P. et al. Local extracellular matrix alignment directs cellular protrusion dynamics and migration through Rac1 and FAK. Integr. Biol. (Camb). 8, 821-835

69. Mould, A. P., Craig, S. E., Byron, S. K., Humphries, M. J. \& Jowitt, T. A. Disruption of integrin-fibronectin complexes by allosteric but not ligand-mimetic inhibitors. Biochem. J. 464, 301 LP-313 (2014).

70. Calvo, F. et al. Mechanotransduction and YAP-dependent matrix remodelling is required for the generation and maintenance of cancer-associated fibroblasts. Nat. Cell Biol. 15, 637-646 (2013). stiffening and immune cell infiltration. Integr. Biol. (Camb). 7, 1120-1134 (2015). 
(2016).

$1142 \quad 73 . \quad$ Broussard, J. A., Webb, D. J. \& Kaverina, I. Asymmetric focal adhesion disassembly in

1143 motile cells. Curr. Opin. Cell Biol. 20, 85-90 (2008).

1144 74. Margadant, C., Monsuur, H. N., Norman, J. C. \& Sonnenberg, A. Mechanisms of integrin 1145 activation and trafficking. Curr. Opin. Cell Biol. 23, 607-614 (2011).

1146 75. Majumdar, R., Sixt, M. \& Parent, C. A. New paradigms in the establishment and maintenance of gradients during directed cell migration. Curr. Opin. Cell Biol. 30, 3340 (2014).

1149 76. Ehrlich, M. et al. Endocytosis by random initiation and stabilization of clathrin-coated pits. Cell 118, 591-605 (2004).

1151 77. Artym, V. V, Yamada, K. M. \& Mueller, S. C. in Extracellular Matrix Protocols: Second

1152 Edition (eds. Even-Ram, S. \& Artym, V.) 211-219 (Humana Press, 2009).

1153 78. Lücker, P. B. et al. A microgroove patterned multiwell cell culture plate for high-

1154 throughput studies of cell alignment. Biotechnol. Bioeng. 111, 2537-2548 (2014).

1155 79. Ray, A. et al. Anisotropic forces from spatially constrained focal adhesions mediate 1156 contact guidance directed cell migration. Nat. Commun. 8, 14923 (2017). 